\title{
LAPURDUTI
}

Euskal ikerketen aldizkaria | Revue d'études basques |

Revista de estudios vascos | Basque studies review

$15 \mid 2011$

Numéro XV

\section{Les contes de Webster : à la découverte du processus de la création}

\author{
Natalia M. Zaïka
}

\section{OpenEdition \\ Journals}

Édition électronique

URL : http://journals.openedition.org/lapurdum/2326

DOI : 10.4000/lapurdum.2326

ISSN : 1965-0655

\section{Éditeur}

IKER

\section{Édition imprimée}

Date de publication : 1 octobre 2011

Pagination : $65-100$

ISSN : 1273-3830

\section{Référence électronique}

Natalia M. Zaïka, «Les contes de Webster : à la découverte du processus de la création », Lapurdum

[En ligne], 15 | 2011, mis en ligne le 15 octobre 2014, consulté le 21 avril 2019. URL : http://

journals.openedition.org/lapurdum/2326 ; DOI : 10.4000/lapurdum.2326 


\title{
Les contes de Webster : à la découverte du processus de la création ${ }^{1}$
}

\author{
Natalia M. ZAÏKA \\ Institut de Recherches Linguistiques de l'Académie des Sciences de la Russie \\ LUniversité d'Etat de Saint-Pétersbourg
}

Létude des manuscrits est avant tout une réflexion sur l'avant et l'après, sur l'ordonnance chronologique des matériaux [Espagne 1988 : 51].

\section{Remarques générales}

Dans notre article, on analysera des questions concernant la textologie et la génétique du texte dans le domaine du folklore basque. Pour cela, on étudiera un manuscrit de Wentworth Webster (rédigé dans les trois langues: français, basque et anglais), ainsi qu'un manuscrit des mêmes contes réécrits par Julien Vinson (rédigé en basque seulement), les deux datant de la fin de $\mathrm{XIX}^{\text {me }}$ siècle ${ }^{2}$. Les premières éditions des contes basques publiés en anglais quelques années après la collecte seront indispensables pour notre recherche. On comparera les variantes du texte basque et sa traduction en français et anglais, en suivant l'histoire textuelle de l'échange des folkloristes.

Parmi les recueils les plus importants des folkloristes habitant au Pays Basque Nord, (Webster, Cerquand et Barbier ; on ne mentionne pas les recueils de Julien Vinson qui sont compilateurs par excellence), celui de Webster est, sans doute, le texte le plus original et authentique. Cette originalité a été conservée dans plusieurs éditions et traductions du texte, grâce auxquelles nous pouvons accéder à des informations assez exactes concernant les contes basques, qui sont indispensables pour les analyses folkloriques. Les autres éditions bilingues sont moins importantes d'un point de vue scientifique (dans celui de Barbier il manque plusieurs types des contes importants pour la tradition folklorique basque et celui de Cerquand souffre d'une traduction souvent incorrecte et littéraire).

Tout d'abord nous ferons quelques remarques textologiques concernant le domaine du folklore basque. Ensuite, nous nous concentrerons sur les problèmes de la collecte des contes, ainsi que les informants de Webster. Puis, nous décrirons les deux manuscrits et analyserons les relations entre eux. Enfin, nous étudierons le fonctionnement du recueil à l'époque moderne.

1. Je tiens à remercier Mme le professeur Aurelia Arkotxa et Joana Pochelu pour leur aide précieuse.

2. Les deux manuscrits se trouvent à la Bibliothèque municipale de Bayonne (MS 88 et MS 720). 
Pour chaque aspect concernant les deux manuscrits, nous avons été obligé d'analyser les textes et leurs notes de bas de page et des notes marginales, différentes éditions des contes, des lettres écrites par les folkloristes et celles qui leur ont été adresées, ainsi que des articles de l'époque où les contes ont été mentionnés.

\section{Remarques textologiques}

Au XIX siècle la textologie devient très importante dans le domaine des sciences humaines. On commence à s'intéresser à l'histoire du texte et à étudier des corrections du texte faites par des copistes. La génétique du texte utilise les méthodes qui ressemblent à celles de la textologie. Si la génétique analyse plutôt le travail d'un seul auteur sur certain texte, la textologie compare le travail des copistes différents, toutes les deux étudiant l'histoire des corrections et de la publication des textes.

Les problèmes que se pose la textologie, d'après un textologue russe, D. S. Likhatchev, sont les suivants : le but de cette science est d'étudier l'histoire du texte manuscrit pendant toute la période où le texte existe, ainsi que ses changements dans toutes les variantes de l'auteur, des copistes, des rédacteurs et des compilateurs. Le textologue doit reproduire très en détail le procédé du travail sur l'œuvre, restituer le processus créatif [2001 : 32, 48]. Jusqu'à maintenant, on connaît peu d'œuvres consacrées aux contes traditionnels d'un point de vue génético-textuel, celles qui envisagent les variantes de la même invariante ou bien les variantes d'un certain texte fini. Dans notre recherche, on essayera d'analyser le folklore basque de ce point de vue.

Le chercheur en folklore devrait se poser la question suivante : est-ce qu'on peut utiliser les méthodes de la génétique du texte dans les œuvres folkloriques, puisque il s'agit de deux procédés tout à fait différents - la création du texte, surtout le texte imprimé, et la conservation du texte. Cependant, il arrive très souvent que les textes folkloriques comprennent les éléments de la création, de l'ornement. Bien que le recueil de Webster soit un objet folklorique, il serait raisonnable de le traiter en utilisant des méthodes de la génétique du texte, puisque dans un conte traditionnel, contrairement aux manuscrits et aux contes publiés, il n'y a pas de hiérarchie des variantes, toutes étant également importantes.

La notion de la variante sera indispensable dans notre analyse des manuscrits. Selon les spécialistes en génétique du texte, « le terme de variante désigne dans les éditions critiques des textes anciens ces possibilités alternatives dont les philologues scrupuleux conservent la mémoire tout en ne leur accordant qu'une validité inférieure à celle du texte principal, finalement retenu » [Espagne 1998 : 1]. Dans notre travail, on utilise la notion de la variante dans un autre sens : pour la textologie du folklore les changements du texte original ont autant d'importance ou parfois plus d'importance que le texte dont il s'agit.

Notre travail textologique a cela de particulier que nous avons seulement deux manuscrits, dont un comporte deux variantes de plusieurs contes en basque, bien que normalement, dans les travaux textologiques et génétiques, on étudie plusieurs variantes (plusieurs brouillons). Cependant, même si l'on imagine que dans le texte de Webster il s'agisse d'un texte idéalement transcrit, il ne faut pas oublier qu'on peut analyser une seule variante parmi les autres, celles que les informants de Webster et d'autres conteurs de leur entourage auraient pu raconter.

Puisque la seule édition des contes basques publiée par Xipri Arbelbide n'est pas tout à fait fidèle au texte, il serait souhaitable que nos recherches textologiques sur le texte des deux manuscrits en question aboutissent à leur publication, qui devrait, comme le dit Lebrave, 
en décrivant des principes d'une bonne édition, associer " d'une manière indissoluble la description, le commentaire et l'interprétation» [Lebrave 2006 : 18]. Ce problème est d'autant plus important qu'il n'existe pas d'éditions fac-similés des contes basques (ce qui serait indispensable de faire à présent pour les manuscrits de Webster et de Vinson). Nous espérons, donc, qu'un jour nous pourrons publier tout le manuscrit dans sa totalité dans les versions imprimées et électroniques, y ajoutant une version fac-similé.

\section{La collecte, les informants et les variantes}

Avant de commencer à analyser les manuscrits en question, nous essayerons de comprendre comment Webster a réussi à collecter tous ses contes. Pour cela, il sera raisonnable d'étudier les remarques dans le manuscrit de Webster et son livre Basque Legends, ainsi que les textes des contes et d'autres documents.

\section{Le procédé de la collecte}

\section{La place du recueil de recueils de Webster dans sa vie scientifique}

Pour mieux comprendre le procédé de la collecte, il faut se poser la question suivante : quelle était l'attitude de Webster par rapport aux textes folkloriques basques qu'il a recueillis?

Après la mort du prêtre en avril 1907 à Sare, G. Lacombe fait paraître au mois de mai de la même année dans la Revue Internationale des Etudes Basques la nécrologie de Webster où il l'appelle «savant modeste et laborieux » qui « venu par hasard dans le Labourd et séduit par le charme étrange de cette contrée, voulut y finir ses jours» [Lacombe 1907 : 323]. Quoique Webster soit une des premières personnes qui ait commencé à recueillir les contes populaires basques, ce fait n'est pas mentionné dans la nécrologie. Pourtant on attribue l'épithète de « remarquable » à un autre livre, Les loisirs d'un étranger au Pays basque [Ibid.]. Nous pouvons en tirer la conclusion suivante: pour Lacombe et quelques-uns de ses contemporains le recueil des contes de Webster n'était pas le plus important parmi ses travaux. En revanche, pour les bascologues modernes le recueil de Webster reste l'un des textes indispensables dans les études folkloriques, de cette façon, selon Xipri Arbelbide « Egin duen lanik interesgarriena da bere euskal ipuin bilduma » (Parmi les œuvres qu'il a fait, son recueil des contes est le plus intéressant) [Webster 1993 : viii].

\section{Les descriptions précédentes du processus de la collecte}

Comme le manuscrit de Webster a été très peu analysé, il est extrêmement difficile de reconstituer tout ce qui est lié à la collecte des contes. Pourtant, dans plusieurs travaux on en trouve quelques raisonnements qui ne semblent pas assez fondés.

Dans son article sur la vie de Webster, P. Urquizu décrit la collecte des contes de la façon suivante: " Ipuinen bilketan lagundu zioten gehienak andreak izan ziren. Badirudi batzuk sutondoan kontatuak zirela eta beste zenbait beste modu honetan bilduak. Lehenik goialdean joaten zen errekan goiti amuarrainak harrapatzen; hauek saskiratuak zituenean herriko apaizaren etxean ate-joka agertzen zen. Horrek oso gogo onez errezibitzen zizkion amuarrainak. Azkenean, biok atsoren batekin ekartzen ziren kafe garaiko solasa haren ipuinekin goxatzera. Websterrek biltzen zituen arkatzez, eta apaizak argitzen komprenitzen ez zituen hitzak» [Urkizu 2000 : 8]. Malheureusement, l'auteur n’indique pas les sources de ces informations. Pourtant, Webster ne parle pas du lieu où les contes ont été racontés, ni de 
ses relations avec le prêtre du village (mais il est reconnaissant à Mme Bellevue pour son aide dans la collecte du texte et la traduction); plusieurs de ses premiers contes ont été écrits à l'encre et non au crayon.

Un autre chercheur qui travaille sur les contes de Webster reconstitue le processus de la collecte en faisant plusieurs suppositions qui contiennent plusieurs fautes (n'indiquant pas non plus la source) [Mercero $2007: 89$ ] :

«Parece que Webster y sus narradoras ${ }^{3 e m e}$ apostaron a favor de la narración en grupo [Effectivement, parfois les contes ont été racontés en présence des auditeurs, mais il est impossible de prouver que cela concernait la plupart des conteurs de Webster - N. Z.]. Los más famosos vascologos que vivían en aquella época en Lapurdi buscaron a gente aficionada a los cuentos y a la narración y la mandaban a la casa Dajieu-baita de Webster [une supposition mal fondée, d'autant plus que c'est Mme Bellevue que Webster remercie pour l'avoir aidé à collecter les contes; il faut ajouter que plusieurs informants de Webster ne venaient pas de Sare où il habitait, et il serait très douteux que les paysans basques aient décidé d'aller dans un autre village simplement pour raconter un conte à Webster — N. Z.].

Reunidos en una habitación [ou peut-être, en plein air ? - N. Z.] y con el rico olor de café extendiéndose por el aire [comme Urquizu, Mercero est certain que Webster aimait le café N. Z.], Webster tenía preparados encima de la mesa de la sala sus folios en blanco y el lápiz [plusieurs brouillons de Webster on été écrits à l'encre - N. Z.].

A su lado se sentaba la señora Bellevue [Webster n'en dit rien dans son livre]. La señora Bellevue que era la proprietaria de Dajieu-baita, imprescindible colaboradora del señor Webster, fue la que le ayudó a trasladar al papel el vivo lenguaje de los lugareños [vu le nombre de fautes que Webster a fait dans les premiers textes, il est plus logique de supposer qu'elle l'a aidé à recopier les brouillons en corrigeant les fautes - N. Z.] .

Los demás, alrededor, formando un semicírculo, en asientos traídos de aquí y allí [encore une invention de l'auteur - N. Z.].

Tras las presentaciones de cortesía, las narradoras empezaban ttuku-ttuku, por turnos, cada una contando lo suyo. En un par de horas finalizaba la sesión, y en una buena tarde, Webster tenía entre ocho y diez cuentos nuevos para su colección [on ne peut pas être sûr que les informants racontaient les contes à tour de rôle ; on ne sait pas quelle était la durée des « séances », est il est très douteux que Webster enregistrait huit ou dix contes à la fois, vu qu'il n'y a que 87 contes dans son manuscrit - N. Z.].

[...] Le contaban cuentos medianos y cortos, sobre todo durante las primeras sesiones, y los cuentos más largos aparecieron durante las últimas sesiones [...] [on ne peut pas affirmer que c'était toujours le cas - N. Z.].

Sin duda Webster hizo un esfuerzo increíble para recopilar todos esos cuentos en un idioma que conocía someramente, y la señora Bellevue tuvo que tener también una paciencia loable, para recopilarlo todo y bien [...].

3. Comme la plupart des informants de Webster étaient des femmes, il s'agit bien de "narratrices" au féminin. 
[Comme on le voit, dans un petit passage d'une moitié de page, le chercheur crée toute une histoire de la collecte sans jamais indiquer les sources - N. Z.].

\section{Les informants et la narration}

\section{La rapidité de la collecte}

Tout d'abord, il faut remarquer que la collecte a été extrêmement rapide. Si Azkue a mis une cinquantaine d'années à recueillir tous ses contes, le savant anglais a recueilli les siens entre 1873 et 1875 (deux contes, très probablement recueillis par Vinson qui l'a aidé sont datés de décembre 1875). Bien que Webster vécut jusqu'à la fin de ses jours à Sare, il n'a plus recueilli, ni publié de textes des contes (et en étant prêtre, il en avait sûrement la possibilité). Probablement, c'est la publication d'un grand nombre de contes basques par Cerquand qui a permis à Webster de cesser ce travail. Cependant, ayant laissé son manuscrit à la Bibliothèque municipale de Bayonne il croyait que quelqu'un pourrait les publier plus tard.

Il convient de mentionner un événement important qui se produisit pendant la collecte. La première partie des contes de Cerquand est publié dans le Bulletin de la Société des Sciences, Lettres et Arts de Pau. Sans doute, le fait que Cerquand ait été le premier à publier les contes basques aurait pu influencer Webster dans sa collecte des contes.

\section{Les gens qui ont aidé Webster à recueillir les contes}

Webster note dans son livre que la collecte a été commencée grâce à Antoine d'Abbadie (« Our first acknowledgments are due to M. d'Abbadie, of Abbadia, the well-known "Membre de l'Institut," for his kind assistance and ready communication of the legends in his possession, and which were the starting point of our work » [Webster 1879: xvi]). C'est lui qui a transmis à Webster une légende de Tartalo : «OUR next story was communicated by M. d'Abbadie to the Société des Sciences et des Arts de Bayonne. The narrator is M. l'Abbé Heguiagaray, the Parish Priest of Esquiule in La Soule » [Ibid. : 4]. Il faut remarquer qu'Antoine d'Abbadie en même temps essayait de recueillir des pièces du folklore basque, comme on le voit dans une lettre où il s'adresse à deux vicaires de Mauléon le 5 janvier 1876, après la publication des premiers contes de Cerquand : "Voilà plus d'un an que j'ai devant moi votre lettre du 26, 7re 1874 et je n'ai rien reçu de vous, ni appris [...] si vous avez travaillé à recueillir les légendes des vieux Basques » [Abbadie 1997: 452].

La personne qui a aidé Webster à recueillir les contes était Mme Bellevue, comme il l'indique dans son livre, en la remerciant : " Madame M. Bellevue, of Dajieu-baita, through whose kind intervention the majority of these tales were collected, and who assisted in the translation of almost all » [Webster 1879: xvi]. On pourrait supposer que la plupart des textes ont été recueillis par Webster. Pourtant, au moins un des contes est raconté à la fille de Webster (les feuilles collées avec une numérotation supplémentaire, sans traduction en français - José Marie Errua).

Dans son livre Basque Legends, Webster atteste l'aide précieuse de Julien Vinson ${ }^{4}$ dans

4. Pour la bibliographie de Vinson voir [Urquijo 1927]. 
ses recherches folkloriques: "I had hoped to have joined the name of M. J. Vinson, the wellknown Basque and Dravidian scholar, to my own as joint-author of this simple work. I should hardly have had the courage to have undertaken it had I not been assured of his invaluable assistance in difficulties about the language of the originals. Unavoidable circumstances have, however, prevented his seeing the Basque of many of the later tales, and he therefore prefers that the "Essay on the Basque Language" should alone bear his name" [Webster 1879 : xvi]. Vinson, dans un article consacré au folklore basque remarque qu'il a « beaucoup couru le Pays basque à la recherche des contes, devinettes, etc. » [Vinson 1884: 4]. De plus, c'est Vinson qui a fourni à Webster la première partie des contes de Cerquand dont le folkloriste anglais parle dans un bulletin en octobre 1875 [Webster 1875: 167].

Quels étaient les liens qui unissaient Webster et Vinson? On a assez peu de témoignages, et aucun article consacré aux relations de ces deux folkloristes. Dans son Folklore du Pays Basque Vinson adresse la dédicace à Webster, qui « may rightly claim to be called the creator of basque folklore » et « the first who intendes to plough the field of euskarian popular tales» [Vinson 1883 : ix], bien qu'il connaisse et utilise les travaux de Cerquand. Vinson écrit dans la préface de son livre: «Although you were so kind as to designate me as a collaborator in your Basque Legends, you know how little I could help you. Yours was all the work, and especially the very difficult one of collecting original texts from the mouths of illiterate countrymen and maidservants, in so hard a language » [Vinson 1883: ix].

De l'autre côté, Vinson écrit qu'un grand nombre de contes qu'il publie « ont été recueillis par M. Webster et par moi [Vinson], à Saint-Jean-de-Luz, principalement, de 1874 à 1876 ». Cela nous permet de supposer que dans le recueil de Webster il y avait des contes recueillis par Vinson. Dans son livre en anglais, Webster indique : "We add another version of this popular tale, collected by M. Vinson from M. Larralde de Lesaca, of St. Pée-sur-Nivelle » (Il s'agit du conte de Jésus Christ et le vieux soldat). On peut également supposer que le conte Ukalicho du manuscrit a été recueilli par Vinson, puisque les deux contes viennent de la même informante (où de deux personnes venant de la même famille et portant le même nom), et on été recueillis le 3 décembre 1875. Pour tous les contes de Webster que Vinson publie dans le Folk-lore du Pays Basque ce dernier indique systématiquement l'année de la collecte (1874, 1875 et une fois 1876) — les informations qui sont absentes dans le manuscrit de Webster, ainsi que dans son livre. Evidemment, ce sont les relations de Vinson avec Webster qui lui ont permis d'indiquer la date.

\section{Les informants}

La plupart des contes du recueil viennent de femmes, ce qui est ordinaire dans la tradition orale. La plupart des contes du recueil ont été racontés par Estefanella Hirigaray, Catherine Elizondo et Laurentine Kopena. Très rarement dans le texte, on trouve une date précise (il peut s'agir des textes recueillis par Vinson). Le village de provenance des conteurs n'est pas indiqué systématiquement. Pour certains conteurs, Webster ne le fait que dans le texte publié, cf. « Estefanella Hirigarray, of Ahetze » [Webster 1879:4]. Plusieurs contes ont été recueillis à Saint-Jean-Pied-de-Port et Guéthary (par exemple, The Serpent in the Wood raconté par Gagna-haurra Hirigaray). Mariño Amyot et Louise Lanusse venaient de Saint-Jean-Pied-dePort [W502, 510], et M. de Betscheller de Baigorry [W368r].

Dans son manuscrit et le livre Basque Legends, Webster indique les conteurs des 61 contes parmi les 85. Dans son livre anglais, Webster en donne une explication: "Some of our tales 
are not signed; we believe these are to be divided between Catherine Elizondo and Laurentine Kopena. Fresh names we think we always put down, but these brought so many tales that we sometimes omitted it with them, and in the rearrangement for printing we have lost our clue" [Ibid. : 217]. On connaît très rarement la profession du conteur (Gachina la fileuse ou Gachina the net-maker dans le livre, l'instituteur Julien Olhasso), encore plus rarement leur âge (Laurentine Kopena a trente-cinq ans et Louise Lanusse a entre soixante-dix et quatrevingt ans; dans un commentaire de son livre, Webster mentionne le conte Arrosa raconté par Estefana Hirigarai et indique l'âge de la conteuse - entre 70 et 80 ans).

D'où viennent les informants de Webster? Il ne le mentionne pas régulièrement dans son manuscrit, pourtant grâce au livre, on peut découvrir certaines choses intéressantes: Ainsi, dans l'introduction de son livre il écrit : "We fear scientific men will be disappointed in this collection. Notwithstanding that we have been careful to collect from those who know the Basque only, or who certainly knew only Basque when they first learnt these tales, yet they are evidently much mixed with French and Spanish" [Ibid. : viii].

Il est bizarre que Webster dans l'édition des contes basques qui est extrêmement bien faite, ne donne que des courtes références sur ceux qui lui ont raconté ces contes. Il est bien possible qu'il ne les connaissait que superficiellement. Tandis que les instituteurs de Cerquand notent le nom de famille des informants (ce qui est assez simple comme ils habitent dans le même village et se connaissent bien), Webster a des difficultés à apprendre le nom de ses conteurs: "The sister of Laurentine. This may be Toutou, but in the Basque country it is sometimes difficult to get hold of a person's surname. "Who is Laurentine?" you ask. "She is Toutou's sister," is the reply. "But who is Toutou?" "She is Laurentine's sister." If you want to get anything more out you have to cross-examine for half-an-hour" [Webster $1879: 217$ ]. Dans le livre anglais, Webster des fois indique les prénoms officiels de conteurs : Mariño Amyot dans le manuscrit devient Louise Amyot dans le livre.

Dans le même livre (il s'agit du conte de Peau d'âne, raconté par Laurentine), on trouve une des rares remarques sur les informants de Webster qu'on voit dans la note de bas de page suivante: "This was explained as meaning "Beaten with the Slipper." This version came from the Cascarrot, or half-gipsy quarter of St. Jean de Luz, and may not be purely Basque. Except in one or two words the language is correct enough - for St. Jean de Luz". Pourtant, on ne sait pas si c'était Laurentine qui venait du quartier en question ou si c'était là-bas qu'elle a appris cette version.

Selon Webster, plusieurs paysans qui lui récitaient les contes ne parlaient pas français, cf. "G. H., the narrator of the above tale, did not know a word of French" [Webster 1879: 157]. Cependant, on ne sait pas, si cela concerne tous les informants (en général, on sait très peut de la transmission des contes, ce qui est caractéristique de beaucoup de recueils de l'époque). On peut supposer que les contes ont été appris aux parents des conteurs qui étaient le plus souvent des basques monolingues, mais dans le manuscrit Webster n'indique que quelques fois les sources du conte (il s'agit de Laurentine qui a appris quelques contes à sa mère et Pierre Bertrand qui a appris un conte à sa grand-mère). Dans la préface de son livre anglais, il fait une observation concernant les contes de Tartaro : « Several of our narrators were utterly ignorant of French, and learnt the tale as children from old people, who died a few years since at upwards of $80 »$ [Ibid. : 3]. Savaient-ils lire et écrire ou pas? Il est probable que certains d'entre eux ne le savaient pas, sinon Webster aurait fait moins de fautes, dans ce cas il aurait pu demander l'orthographe des mots inconnus. 


\section{La transmission des contes}

A la fin du XIXème siècle, les folkloristes notent très rarement les conditions dans lesquelles les narrateurs ont raconté leurs contes. C'est le cas du recueil de Webster. Au moins, plusieurs contes ont été racontés en présence des auditeurs. Ainsi, Webster remarque dans une note de bas de page de son livre: "At an exclamation of surprise from one of the auditors, the narrator piously said, "It is the Holy Virgin who permitted all that." [Ibid. : 162] (cf. [W575] " C'est la Ste Vierge qui permettait tout cela »). Webster fait également une autre remarque sur un des contes: "One of those present here interrupted the reciter — "What did she hit the serpent on the tail for?" "Why, to kill him, of course," was the reply; "ask Mr. Webster if serpents are not killed by hitting them on the tail?" [Ibid. : 32]

Parfois, les commentaires du manuscrit sont plus brefs : "On demandait à la conteuse Le roi ne faisait pas la fête chez lui? Elle répondit : Non c'était à la mairie ». Cette réplique prouve que pendant la récitation des contes Webster posait des questions aux conteurs. Dans son livre Webster donne un commentaire plus élargi: "The narrator was here asked "if the place of the dance was at the king's palace." "No," she gravely replied, "it was at the mairie." In other tales it is on the "place," i.e., the open square or market-place which there is in most French towns and villages in the south. It is generally in front either of the church or of the mairie". Il aurait pu exister encore un brouillon, où Webster l'indique.

Parfois Webster fait des observations sur le processus de la transmission des contes : "When we hear these tales related by peasants ignorant of French, we may still ask how far they have learnt them at second or third hand from the printed works, and how far they are reciting the crude materials out of which those works were originally composed ? This is a question which can only be fully answered when all the legends in all the languages and patois of France shall have been collected and compared. Meanwhile, we beg our readers to accept these few tales as a small and not very valuable stone contributed towards the erection of so vast an edifice » [Webster $1879: 58]^{6}$. A la fin de l'année 1875, Webster écrit dans un article consacré aux contes basque de Cerquand : « on les débite [les contes] pendant qu'on épluche le maïs pendant l'hiver » [Webster $1875:$ 173].

Dans certains cas, Webster arrive à suivre les processus de la transmission du conte plus exactement, mais cela reste plutôt une exception : "We have also, in Basque, a version of Madame d'Aulnoy's "Abenan." It seems to be a mixture of various legends strung together by this fanciful writer"; "This tale, though without doubt derived from the French, we can trace up in Basque further than any other. It was told us by a lady of between seventy and eighty, who heard it as a child from an old nurse, whom she distinctly remembers to have told her that she learnt it as a child from her mother. It must thus have existed in Basque over a century" (il s'agit d'un conte recité par Louise Lanusse) [Webster 1879 : 191].

5. Nous indiquons le manuscrit de Webster avec la lettre W (r pour le recto, $v$ pour le verso) et celui de Vinson avec la lettre $\mathrm{V}$.

6. Il faut ajouter que le travail dont Webster parle a été effectué par Delarue et Tenèze, plus d'un siècle plus tard, et c'est maintenant qu'on commence et continue à créer de nombreux recueils des contes, surtout dans les pays européens. 


\section{La narration}

Parfois les narrateurs produisent des réflexions concernant leurs contes. En comparant le texte publié dans Basque Legends et les versions du manuscrits, on trouve dans le livre certaines remarques qui sont absentes dans le manuscrit ( This doubling of a price is to get a thing more quickly done - in half the usual time. At least, that was the narrator's explanation » [W100]). Une autre remarque du livre de contes en anglais qui concerne la collecte n'est pas non plus indiqué ni dans le texte basque, ni dans le texte français (« This Fleur-de-lys was supposed by our narrator to be some mark tattooed or impressed upon the breast of all kings' sons » [Webster 1879 : 24]). Il est possible qu'il ait existé un autre brouillon, probablement en anglais, où Webster a noté cette remarque.

Dans ses notes de bas de page, Webster analyse différents types de narrations: "In the legends they are reciting a text learnt by heart... But they tell their stories of witchcraft in their own words, just as they would narrate any other facts which they supposed had happened to themselves or to their neighbours » [Ibid. : 64].

Normalement, une manière différente est typique à chaque conteur particulier (il s'agit surtout du style des contes), les uns ayant tendance à raconter de longs contes, et les autres plus courts. Nous constatons que les diverses conteurs préfèrent raconter des contes de types différents. Ainsi, Estefana Hirigaray préfère plutôt des contes courts, tandis que Laurentine Kopena en raconte de plus longs et plutôt merveilleux, qui trouvent des correspondances dans le folklore européen. Les contes de Laurentine ont plus de digressions. A titre d'exemple on cite un extrait du conte Errege eta bere hiru alabak (après que la fille accepte d'épouser le serpent) :

« Azorrek behar duela gan bere aitaen etcheat minzatzeat aphezak eta behar diren gauza guzien preparatzeat Biharamunian izaen direla espos Badoha neschatcha erran bezala erraten dio aitari ezkonzen dela Sugéakin beharamunian eta eyan denak prestatuko tuen hartako Aitak erraten dio Baietz Bañian pena zuen Aizpek galditzen diote ere noekin den espos eta arritubak dire aditziaikin Sugeaekin iñ behar duela Fifine badoha bere etcheat » qui n'ajoute rien au contenu du conte (dans d'autres contes, on peut lire tout simplement qu'on a invité le père de la fille).

On va citer encore un long fin d'un conte Errege eta bere hiru alabak raconté par Laurentine : « Badohazi biaramunian ungi yostatzen dire badohazi beren jaureguiat bere gauza ederren biła ez beitzuten geihago nahi mendi zoko hartan kargatzen tuzte karruak eta charretak gauza baliyosez eta badohazi erregeen etcheat han bizitzeat Andre gozte harek beititu lau haur bi mutilak eta bi neschka Aizpek d/j/elosia handia beitzuten aitak egorri etchetikan kampoat erregek eman ziyoen bere korona bere suyiari yadaneko errege semea zen Ungi bizi baziren, ungi hil ziren ».

Dans le conte Amatchi lamiñaen erregiña raconté par Laurentine, on voit encore une digression : "Ezkonzek eguna ethorri zenian Erregiña arribatu zen ekharri zuen espos eguneko arropa diamantezkoa Biharamueko urezkoa eta hirugarren eguneko ziłarezkoa Pensaazue zenian ederra zen bere bista diamantaekin eta arropa diamantaekin ezin beira zitzaioen Amatchik eran zigoen han izaen zituela bere koñatak Banian ez heyien beldur izateko etzireela urbiltzen haren edertasunari ilki zenian espos egunian ezin beiratu zioten bere koñatek hala distiraatzen zuen Erran zuten elkharri Arrozoña zuen potzoak errateko edera zela andre hura hiru egunez pasaiya tuzuen Bellaros eta mundu guzia soratua zen haren edertasunez Amatchik bestak pasatu eta gan zen bere etcheat Arrosa etzuen nahikatu kitatu Bellaros Amatchik erran 
zigoen Bellarosi Nola buraso pobretaik atheratzen zen harek lagundu zituztela demboraz Bañian gar gehiago akabatuak zituztela harek emanak Bellarosek Eman zioten bizitzekoa ausarki deneri Berak izan zituen laur haur bi mutil eta bi neschka eta ungi bizi izan baziren ungi hil ziren".

Laurentine ajoute des fins atypiques aux contes typiquement merveilleux, tels que Peau d'âne: "bizi ziren hañitz urrus zembeit demboraen buruban izan zituzten bi haur mutil bat eta neschka bat zazpi urte in zutenian zaharrenak hil zen erraten ziotelaik bere aita eta amari Zeruat zuala hekien plaza egiteat Zortzi egunen buruan hilzen zaiyote bertze haurra ere eta erraten diote harek ere zeruat duala eta tokia prest atchitiko diotela hekieri ethorriko direla laster hekien ganat eta erran bezala urte baten buruban yustu yustuba hil ziren jaun-andreak eta gan zeruat jaun andreak".

Le plus souvent le héros des contes de Webster est appelé «mutiko » ou « jaun gazte » (« andre gazte »). Parfois les informants préfèrent certains prénoms. Ainsi, Laurentine choisit le prénom Fidel pour l'héroïne du conte de Peau d'âne, ainsi que pour le héros d'Ezkabi-Fidel. On emploie le prénom Fifine dans deux contes (Errege eta hiru alabak /La belle et la bête et Zoria /Le Pou). Certains noms sont insérés au milieu du conte (c'est surtout typique des contes de Laurentine, cf. Marie-Madeleine, Fifine et Azor). On peut se demander pourquoi on trouve le même nom (Yaundeko) dans deux contes de Webster, tandis qu'il est absent dans les autres recueils basques. Il est possible qu'il ait demandé à son informant s'il connaissait le conte de Yaundeko.

\section{Le contenu des textes}

Dans son manuscrit, Webster recopie toutes les variantes, qu'il a notées, mêmes inédites, tandis que dans les recueils de Cerquand et Barbier plusieurs variantes ont été mentionnées, mais n'ont pas été notées. Cependant, s'il s'agit d'un conte qui a beaucoup de variantes, Webster en note moins qu'on ne lui en raconte, ce que prouvent plusieurs extraits de son livre Basque Legends : "We heard, also, another version, which, unfortunately, we did not take down » [Webster $1879: 177$ ] ; "Though collected mainly in the neighbourhood of St. Jean de Luz, we have tested them by enquiry of natives of all the provinces, and find that they are equally well known in La Soule and in Basse Navarre as in the Labourd. We never met with a Basque peasant who could not tell us what are the Tartaro, the Heren-Suge, Basa-Jaun, and the Lamiñak» [Ibid. : viii]. En parlant des variations de Peau d'Ane, Webster remarque: «We have four other variations of the above story, written down, with others, that we heard, but did not copy out » [Ibid. : 165].

Bizarrement, dans le manuscrit on ne trouve pas de textes étiologiques, des mythes et des légendes au sens propre du mot (telles que les contes de laminas), bien que Webster affirme dans ses notes de bas de pages qu'ils sont courants. Dans le recueil de Webster, on ne trouve que peu de contes de laminas, où elles ressemblent plutôt aux fées européennes. Cependant, dans un commentaire sur le conte de lamina en couches (traduit à partir du conte 35 de Cerquand), Vinson remarque «M. Webster a entendu raconter à Osse une variante de ce conte. La sage-femme avait été prise à Bedous, et la grotte où on l'avait conduite avoisinait Accous. Elle avait emporté un petit morceau de pain ; aussi, quand elle arriva chez elle, ne trouva-t-elle plus que des briques et des pierres au lieu d'or et des pierreries » [Vinson 1883 : 381]. Évidemment, dans ce conte il s'agit du Béarn voisin, mais c'est étonnant que Webster, ayant entendu ce genre de contes en Béarn, n'en ait pas noté au Pays Basque. 
Dans son article sur les contes de Cerquand, Webster remarque que « les Paraboles ou Allégories sont très répandues parmi les basques » [Webster 1875 : 168]. Pourtant, on n'en trouve pas dans son recueil (elles sont également présentes dans les recueils de Barbier et Azkue)».

Nous pouvons donner plusieurs explications probables pour l'absence de certains genres de contes. Premièrement, il est possible qu'il n'y en ait pas. Comme Webster recueillit et transcrivit les contes d'une partie limitée du Pays Basque (principalement, les contes labourdins), nous pouvons supposer que dans le folklore traditionnel de cette partie du Pays Basque il n'a pas existé ce genre des contes ou il n'en reste plus. Cependant, cette hypothèse nous semble extrêmement douteuse, parce qu'on trouve les textes de ce genre dans les régions voisins et on sait parfaitement que les emprunts entre les territoires voisins sont très fréquents, même s'il s'agit de deux langues différentes. Il nous reste encore une hypothèse : Peut-être que Webster n'était pas intéressé par ce type de textes qui ne sont pas des «contes populaires » dans le propre sens du mot, ni les « légendes » (« legends » en anglais) non plus. Ainsi, on se demande pourquoi on ne trouve pas de paraboles de Jésus Christ et Saint Pierre dans les recueils de Webster, bien qu'en analysant les premiers contes publiés par Cerquand il remarque que ce genre est très répandu parmi les basques [Webster 1875 : 168]. Il est possible que du point de vue de Webster ces textes ne soient pas des «légendes », comme l'indique le titre de son livre Basque Legends. Il s'agit souvent de textes étiologiques qui expliquent l'introduction des objets (les ponts, les églises) ce qui ne doit pas être tellement intéressant pour le lecteur anglophone (et c'est justement le lecteur anglophone qui appréciera une partie de ces contes pour la première fois, pour les autres, il faudra attendre presque un siècle).

Webster se rend parfaitement compte de ce qu'il existe d'autres types de récits populaires qu'il n'a pas recueillis. Ainsi, il témoigne d'avoir entendu des contes sur Roland au Pays Basque, bien qu'on n'en trouve pas dans son manuscrit : «Un paysan Basque-Espagnol m'a égaye pendant quelques heures de marche, entre les Aldudes et Roncesvaux, en me racontant toute l'histoire d'après "la chanson de Roland". Il me désignait tous les endroits, et me décrivait les péripéties de la bataille, et me demandait si j'avais jamais vu cela dans les livre imprimés. Lui, il l'avait appris de son père, qui le racontait pendant les longues veillées d'hiver » [Webster 1875: 172].

Dans le manuscrit de Webster, il y a beaucoup de contes longs. Les contes merveilleux de Webster sont généralement plus longs, que ceux d'Azkue, Cerquand et Barbier ; ils comportent souvent plusieurs mouvements. Le lexique du manuscrit est assez restreint par rapport à celui de Cerquand et Azkue. Parmi les contes merveilleux, il y a certains qui ont les traces évidentes d'emprunts au niveau lexical. Ainsi, en parlant du conte Yaundeko, Webster remarque: "This name was written thus phonetically from the Basque, and it was not till I saw the Gaelic tale that it struck me that it is simply "Jean d'Ecosse" — "John of Scotland", or "Scotch John" [...] This seems decisive that in some way the Basques have borrowed this tale from the Kelts since their occupation of the Hebrides" [Webster 1879 : 146]. On ne sait pas exactement quelles étaient les sources des contes merveilleux dans le manuscrit. Le texte de Perrault est trop long pour que les conteurs ou leurs aieux puissent s'en servir. On peut supposer qu'ils utilisaient plutôt des livres de colportage. 


\section{La description de deux manuscrits et les relations entre eux}

En étudiant les deux manuscrits d'un point de vue textologique, essayons de décrire les modifications qu'on y trouve et de les expliquer.

\section{La description du manuscrit de Webster}

Le manuscrit du recueil Basque Legends de Wentworth Webster quise trouve à la Bibliothèque municipale de Bayonne et qui contient 87 contes en basque et plusieurs traductions est sans aucun doute l'un des textes les plus intéressants pour le chercheur concernant le folklore basque. Il est d'autant plus précieux qu'on ne sait rien sur les manuscrits d'autres recueils de contes basques et que c'est un texte écrit extrêmement long qui inclut les brouillons, ainsi que les textes déjà corrigés et prêts à être publiés. Il existe d'autres manuscrits de Webster concernant l'histoire, les contes et la poésie des Basques qui se trouvent à la Bodleian Library (l'Université d'Oxford) et qui ont été remis à cette bibliothèque en 1921.

Le manuscrit de Webster contient 654 pages de textes basques, français et anglais. La numérotation de recto et verso n'est pas toujours cohérente : certaines pages sont numérotés des deux cotés, tandis que les autres d'une seule.

Le manuscrit, tel qu'il est présenté à la bibliothèque est très hétérogène. Il contient plusieurs cahiers, mais le folkloriste conserve une seule numérotation des pages pour tout le manuscrit (très probablement, ajoutée plus tard). Mis à part les cahiers, le manuscrit contient quatre pages qui ont leur propre numérotation (de 1 à 4) fournissant quelques explications à propos des contes et qui nous donnent une information importante sur les sources des contes et de la traduction : «Les cahiers M.S.S. des Legendes Basque [sic] ecrites par Rev Wentworth Webster à Dajieubaita, Sㅁt Jean de Luz, dans les années $1873-1875$ pour la plupart à la dictée de $\mathrm{M}^{\underline{m} \text { e }}$ Bellevue, la Propriétaire de Dajieubaita. Les traductions Françaises aussi furent faites par elle à vive voix et écrites par M. Webster » [W01]. Comme la plupart des contes ont été racontés par des paysans basques, nous pouvons supposer que Mme Bellevue a lu les brouillons à voix haute. Webster a travaillé sur le manuscrit après la publication de Basque Legends (il indique les contes publiés dans la table de matières). Dans ces pages Webster indique également quels contes ont été utilisés par Julien Vinson dans ses recueils et fait une remarque sur le rôle de celui-ci dans le manuscrit : "Les corrections faites au Basque de M. S. de ces contes sont de la main de M. Vinson» [W04] (pourtant, la plupart des textes basques (sauf le premier cahier) ne sont pas corrigés par Vinson).

Dans le manuscrit, on retrouve encore quelques feuilles qui commencent une nouvelle numérotation (de 1 à 6 , il y en a plusieurs pages vides). C'est une liste de contes manuscrits avec quelques indications du caractère comparatif sur leur édition et leur traduction.

Les feuilles du manuscrit de Webster appartiennent à deux périodes. Le texte du premier cahier est à l'encre en basque (à droite) et en anglais (à gauche) et comporte les pages 1-192 (193). Sur la page 141, on voit une remarque "Text in A / Trans in H », ce qui indique très probablement, la numérotation originale du folkloriste (si Webster utilisait l'ordre alphabétique, il devait avoir beaucoup de cahiers). Dans le premier cahier, les textes basques sont déjà corrigés. Le deuxième cahier est de petit format. Les textes sont au crayon et à l'encre, parfois traduits, quelques textes sont corrigés par Julien Vinson (on reconnaît facilement son écriture). Il contient les pages 193-252. Dans le deuxième cahier on voit les mêmes contes que dans le premier, mais avec plus de fautes. Webster commence ses cahiers par des contes courts (probablement, pour mieux apprendre la langue). Le premier et le deuxième cahier ont 
des dimensions différentes par rapport aux autres cahiers. En revanche, le troisième cahier, les pages 253-623 représentent plusieurs cahiers de la même taille.

La troisième partie des textes, la plus grande, est un brouillon avec la traduction en français faite au crayon et à l'encre (à partir de la page 396 - presque tout à l'encre, avant au crayon). Parfois le texte écrit à l'encre couvre le brouillon fait au crayon. Dans la partie du manuscrit qui est en basque et en français il y a beaucoup de corrections concernant la grammaire de la langue basque. Par contre, dans la première partie les corrections sont plutôt orthographiques. En regardant l'encre, on peut noter que Webster écrivait les contes en basque en entier, et puis la traduction en français, il ne les écrivait pas page par page (cf. [W433]). Le manuscrit contient également un autre cahier (les pages 624-654), où Webster transcrit des textes autres que des contes.

D'un point de vue textologique, c'est le deuxième cahier qui est le plus intéressant et le plus important parce qu'il est le plus ancien. Dans les contes des cahiers suivants il y a moins d'erreurs. Les cahiers de la troisième partie ne sont pas numérotés. La plupart des textes sont de très grande qualité, comme ce recueil a été donné par Webster à la bibliothèque.

Dans le manuscrit de Webster, la plupart des textes n'existent pas en version anglaise. Cela donne à penser qu'il y avait d'autres cahiers qu'on ne possède pas. Cependant, c'est Webster même qui a donné le texte à la bibliothèque de Bayonne après l'avoir fait paraître (on remarque de numéros des pages du livre imprimé dans les cahiers). Pour les folkloristes, l'endroit où se trouvent les autres brouillons en anglais reste un mystère (il serait nécessaire de consulter les archives des bibliothèques en Angleterre). On pourrait supposer que le folkloriste a utilisé le texte anglais pour éditer son livre, de cette façon, il ne le possédait plus lorsqu'il a remit le manuscrit à la bibliothèque.

La partie anglaise du manuscrit de Webster est dans une certaine mesure incomplète. Plusieurs contes qui sont publiés en anglais dans Basque Legends n'y figurent pas. Évidemment, ils ont été traduits plus tard par Webster lui-même à partir de la version française, bien que parfois le folkloriste se serve de la version basque. Ainsi, dans le conte Basa-Jauna un des frères appelle un autre « asto zaharra » [W13], ce qui est traduit à tort en français « tu es un vieillard toi » [W205]. Dans le texte anglais « you are an old man »; les mots « ass » et « donkey » sont écrits au-dessus, au crayon [W12], d'où vient le texte du livre Basque Legends "you are an old donkey » [Webster 1879 : 49]. Les contes traduits en anglais dans le manuscrit sont assez courts et, le plus souvent, moins répandus. Dans le texte anglais de Webster on ne trouve pas de variantes du même conte.

Il faut souligner que le premier texte de Webster qu'on trouve dans la Bibliothèque municipale de Bayonne n'est pas le premier texte de contes que Webster a transcrit. Très probablement, il y avait d'autres brouillons que Webster ne croyait pas nécessaire de laisser à la bibliothèque. Mais d'un point de vue textologique il est important que l'histoire de tous les cahiers comprend très peu de temps (pour les textes modernes c'est souvent le cas, mais pour les textes qui datent du XIII-XVII ${ }^{\text {emes }}$ siècles, par exemple, les textes hagiographiques anciens russes, la période des changements peut encadrer quelques décennies, voire, siècles).

A part l'écriture manuscrite de Webster, et celle de Vinson, on peut trouver encore plusieurs échantillons d'écriture dans le manuscrit (cf. aussi les corrections [W57, 366]). Deux contes sont écrits sous la dictée de Julien Olhasso, instituteur à Bidarray dont l'orthographe est différente et l'écriture l'est aussi légèrement. Le conte de Belzunce n'a pas non plus été écrit par Webster. 
Mis à part les contes, le manuscrit de Webster contient quelque remarques (il cite certains d'entre eux dans son livre Basque Legends ) :

«On trouve la Belle et la bête raconté comme une histoire de Tartaro » [W308r].

«cf. Le vieux soldat et Caballero » [W373v].

«On dit que le héros est idiot » [W394r].

« Jean d'Ecosse » [W399v].

«l'ame est le renard » [W417v].

« see Grimm tale La gardienne d'oies prés de la fontaine » [W420v].

«The fox talks like a little child» [W462r].

« aizpa entre sœurs /arreba avec les frères » [W486r].

« Pour les soins on lave les pieds dans le même chaudron selon le rang " [W488v].

«Sa mère a vu la maison de la jeune fille et le corps de la jeune fille à la chapelle à SaintSauveur »[W492r]

«cf. Aladdin's lamp et la Tabakièra » [W517v]

«On raconte aussi une variante du Pont de Licq de M. Cerquand. On doit deviner le nom du diable. P. Bellevue $»^{7}[$ W521r]

«Livre des contes français» [W538v].

A la fin du conte raconté par Laurentine Kopena on trouve un texte raturé : " Colé receveur /M. Colé buraliste à côté de M Harriette » [W591r]. Probablement, il s'agit des écouteurs du conte.

« autrefois on laissait les enfants 7 ans en nourrice — et pour le premier dans une chambre obscure »[W602v].

"The only tale where the king's son is called Prince » [W613v].

A la page [W127] on voit la remarque suivante : « L. J. V. pretés à M. H. de Meurville 12/3/79 », où il s'agit, très probablement, de Julien Vinson.

On remarque que la grammaire du manuscrit de Webster est assez homogène. Les informants utilisent le solécisme de la côte (cf. [Lafitte 2001 : 296]) (en plus, il y a très peu de textes écrits où l'on trouve ce phénomène grammatical). Dans le texte du manuscrit de Webster la syntaxe est simple, il y a peu de propositions subordonnées. Il est possible que le style des phrases courtes s'explique par le fait qu'au début de sa carrière folkloriste, Webster comprenait très peu le basque.

Les principes de la transcription de Webster sont liés au fait que il ne maîtrise pas très bien le basque. Son orthographe est très proche de l'orthographe moderne, mais dans certains points elle s'approche de la prononciation. En choisissant entre le c et le k, dans la plupart des cas, le savant utilise le $\mathrm{k}$. Les sibilants s/ts et z/tz sont le mêmes que pour l'orthographe moderne et les ch/tch sont différents (ils sont utilisés au lieu de x/tx modernes, ce qui était normal à l'époque). Bien que Webster note assez fidèlement la phonétique, il confond souvent les sibilants (« usten » au lieu de « uzten » [W602r], « guztu» au lieu de « gustu » [W608r], atcho au lieu de atso [W167], etc.), ce qui peut également être lié aux dialectes des conteurs.

7. P. Bellevue pourrait être le fils de Mme Bellevue, qui était abbé. 


\section{Le destin du manuscrit et la préparation des éditions}

En 1902, Webster écrit qu'il voudrait faire une nouvelle édition de Basque Legends, s'il avait plus de forces : "Si después de mi muerte quiere alguien hacer una edición francesa de mi libro, encontrará los materiales preparados en la Biblioteca municipal de Bayona, á donde irán los cuadernos manuscritos que tengo reunidos, tomándolos escrupulosamente de la versión oral » [Echegaray $1908: 374]$.

Le folkloriste a laissé les manuscrits des contes à la bibliothèque à la bibliothèque à la fin de sa vie (bien qu'il ait pu le faire plus tôt), comme on le voit dans la lettre de C. Léon Hiriart, bibliothécaire-archiviste de Bayonne, qui date de 20-9-06, où il mentionne « I vol. in 4 de cahiers M. SS. de Basque Legends ». On ne sait pas s'il voulait ajouter d'autres contes dans la nouvelle édition et s'il croyait nécessaire de publier d'autres contes en anglais. Dans les documents que l'on trouve, Webster ne parle pas d'une autre édition anglaise avec d'autres contes (probablement, parce que le manuscrit se trouve en France). Dans sa lettre, Webster remarque que les textes sont déjà prêts à être publiés. Webster parle de son manuscrit à son ami Julio de Urquijo, qui le note dans un article [Urquijo 1935 : 209]. On va reproduire la partie de la lettre concernant le manuscrit en question qu'il a écrit quelques jours avant de sa mort, le 19 mars 1907 (cette remarque nous sera également utile pour arriver à comprendre les compétences linguistiques de Webster):

« a Volume in $\mathrm{qt}^{\text {deo }}$ containing the cahiers in which I wrote down, often in pencil, my Basque Legends, with the French translation: also a little Basque Comedy performed by the young girls of the sisters school at Sare, and several other pieces of Folk-Lore.

The greater part of this [sic!] Folk lore tales were recited to me by Madame Bellevue ${ }^{8}$, of Dajieu-baita, St Jean de Luz, where I was then leaving as her tenant in the larger house, and she in the smaller one. Her son, l'Abbé Bellevue, now Curé of Hendaye, was then my much loved pupil for Latin and English. I wrote down the Basque phonetically as it sounded to me, not grammatically; and Mde Belleve (sic) supplied the translations afterwards. I did not understand all the Basque; but I could then follow the general sense of a tale or a conversation. Since, especially after I became deaf, I have forgotten the little I knew ».

Malgré cette remarque quelque peu péjorative, à la fin de sa vie Webster s'intéresse constamment à la langue basque dont on voit des preuves dans ces lettres et celles de ses correspondants. Probablement, sa surdité que Webster mentionne l'a empêché de recueillir d'autres contes, mais elle ne pouvait pas l'empêcher de préparer une autre édition plus complète des contes basques en anglais. Cela donne à penser qu'il croyait la deuxième édition des contes suffisante.

Dans la même lettre, Webster déclare son envie d'envoyer encore quelques manuscrits à la Bibliothèque de Bayonne : "I have other cahiers and note books on metters concerning this country, which I shall probably present later to the Bibliothèque de la ville de Bayonne ». On ne sait pas où se trouvent ces textes, mais ils pourraient être liés au folklore basque.

Avant de laisser le manuscrit à la bibliothèque de Bayonne, Webster lui-même a essayé de publier le recueil des contes en français. Pour cela, il s'est adressé à l'éditeur de son livre en anglais, W. Walbrook, qui lui répond le 24 avril 1894 : « As regards giving permission to

8. Probablement, il s'agit de son aide dans la transcription et les traductions. 
M. E. de Bidegaray to translate and illustrate your « Basque Legends » into French I willingly acquiesce; but I would like that Mr. Fielding should express his feelings in the matter. For this purpose I am forwarding copy of your letter and copy of this one to Switzerland to him » [Bonaparte 1934 : 325].

M. de Bidegaray, qui était évidemment chargé de la traduction, annonce le début de son travail dans une courte lettre datant du 7 mai 1894 (quelques jours après la lettre de Walbrook!), où il parle de deux éditions de Basque Legends et écrit : « I begin to day [sic!] my translation and shall send to my brother two or three legends at a time. As soon as he sends me back the text and the illustrations I shall communicate you the whole » [Bonaparte 1934 : 326]. On peut, donc, conclure que Webster était sûr de pouvoir publier les contes. Pourtant, l'édition française n'a pas été réalisée. Une autre question se pose : tout en ayant le texte en français dans ces cahiers (il a traduit le texte en anglais à partir du texte français), pourquoi a-til demandé de traduire les contes encore une fois? Probablement qu'à cette époque, il n'avait que des brouillons de contes.

\section{La description du manuscrit de Vinson}

Le manuscrit de Vinson nous donne des nouvelles pistes pour la compréhension de celui de Webster. Grâce à ce manuscrit, on peut comprendre les processus textuels qui suivaient les changements dans le texte du folkloriste anglais. On a beaucoup de chance d'avoir le texte de Vinson qui a été acheté par la Bibliothèque municipale de Bayonne très récemment, car les manuscrits de contes ne sont pas toujours accessibles. Il est intéressant de remarquer que bien qu'étant secondaire, ce manuscrit est indiqué sur le site du Patrimoine des bibliothèques d'Aquitaine ${ }^{9}$, tandis que l'original de Webster n'y est pas mentionné. Les folkloristes basques et français on beaucoup de chance de pouvoir consulter les deux manuscrits de contes traditionnels, car dans beaucoup de pays il n'en reste point (ainsi, Boggs remarque qu'il ne connaît aucune grande collection de contes de fées manuscrits en Espagne [Boggs 1930 : 7]). Le manuscrit de Vinson a été fait à Bayonne. A ce jour, il est inconnu aux folkloristes. Les textes de contes sont de très haute qualité, il s'agit d'un manuscrit tout prêt à être édité.

Le manuscrit a été acheté par la Bibliothèque municipale de Bayonne en 2005 (MS720). C'est un texte de 284 pages, qui comporte 87 textes de contes, dont 84 sont tirés du manuscrit de Webster (les contes XX, XXI, LXXXVIII ne le sont pas). Outre la numérotation ordinaire, dans le manuscrit de Vinson les feuilles sont numérotées par quatre, le papier n'est pas ligné. Le texte a été écrit sur du papier jaune (il a probablement jauni avec le temps), à l'encre noire. Les pages sont numérotées par Vinson, de la même encre. Les pages du manuscrit de Vinson contiennent quelques fautes de numérotation : le nombre LXXXII figure 2 fois (une fois pour les variantes du conte Tartaro); Au lieu des contes XXXIII et XXXIV nous trouvons un seul conte, il n'y a pas de contes XXXVIII, LXVIII.

Les principes de l'écriture de Vinson ressemblaient beaucoup à ceux de Webster. Dans quasiment tout le manuscrit, l'orthographe est plutôt moderne, bien qu'on y trouve plusieurs textes avec d'autres principes d'orthographe, plus ancienne; Vinson réécrit les textes de

9. http://arpel.aquitaine.fr/frab/document.php?document=2276. 
Webster en conservant cette orthographe (le c au lieu du k, le ç au lieu du z), mais dans deux textes de l'instituteur Julien Olhasso l'orthograhe des deux folkloristes est différente : « Acheria errepostu ematen dio: zure pagamendutzat erranen dausquitzut hirur eguia handiak » [W620v] vs. « Acheriac errepostu emaiten dio: çure pagamendutçat erranen dausquitçut hirour eguia handiac » [V265]. Vinson omet souvent le $\mathrm{r}$ intervocalique qui est présent dans les textes de Webster (errepustarik [W135] vs. errepustaik [V45], hura [W212r] vs. hua [W69], haren [W341] vs. hain [V83]). Il marque certains traits phonétiques dialectaux (ziguen [W309r] vs. zioen, [W54], ematen [W157] vs. emaiten [V78], etchean [W254] etchian [W73]). On trouve aussi des corrections phonétiques qui sont liés aux dialectes: itsasuain [V92] vs. itxasuaen [W360r]. Les consonnes aspirées sont d'habitude marquées (deithua [V1], artho [V5], ikhusten [V6], urhe [V21], erhestun [V51]). La ponctuation est traditionnelle.

\section{La source des contes basques publiés dans un recueil de Vinson}

Il est probable que Vinson traduisait les textes pour son livre célèbre Le Folklore du Pays Basque publié en 1883 à partir du manuscrit MS720 de la Bibliothèque municipale de Bayonne. Les contes de Webster qu'on peut trouver dans le livre de Vinson sont légèrement modifiés ( J'ai naturellement traduit directement sur le texte basque ; dans quelques contes, déjà publiés par $\mathrm{M}$. Webster, j’ai conservé certains détails que mon savant collaborateur avait cru devoir supprimer» [Vinson 1883: XIV]). Dans son manuscrit, Webster mentionne les contes que Vinson a traduits en français, d'où nous pouvons tirer la conclusion que Webster a travaillé avec son manuscrit après la publication du livre de Vinson en 1883. Dans son livre, Vinson préfère traduire les textes de l'original basque lui-même (Vinson a la même attitude par rapport aux autres éditions bilingues, parmi lesquelles on peut citer celle de Cerquand, toujours préférant travailler avec le texte original). Dans son livre, la traduction devient moins littérale, plus littéraire. Ainsi, si dans le cahier de Webster, on calque la phrase basque « asko munduan bezala » comme « comme beaucoup dans le monde », Vinson donne une traduction plus correcte d'un point de vue stylistique : « comme bien souvent dans le monde ». Pourtant, il n’introduit jamais aucun détail supplémentaire.

Sauf ce genre de corrections, Vinson corrige légèrement le style des contes en ce qui concerne l'emploi des temps. Si Webster conserve le présent et le passé de ses locuteurs, Vinson préfère utiliser le passé simple dans son texte : « egun batez heldu zaio yaun bat, eta erraten dio » [W465r], « il lui vient un monsieur qu'il lui dit » [W464v], « and a gentleman comes to him, and says » [Webster 1879: 77] et « il lui arriva un monsieur et lui dit » [Vinson 1883 : 80]. Julien Vinson divise également le texte en alinéa, tandis que les contes chez Webster ne sont pas divisés. Puisque la version de Vinson se veut plus littéraire, il introduit les antécédents des pronoms au cas où l'on trouve une ambiguité : « ematen dio diru hainitz » [W465r], « il lui donne donc beaucoup d'argent » [W464v], « He gives him, then, a great deal of money » [Webster 1879: 77] et « Le nouveau venu lui donne beaucoup d'argent » [Vinson $1883: 80]$.

\section{Les relations entre les deux manuscrits}

La plupart des contes dans les deux manuscrits en question (85) coïncident. Quelles étaient, donc, les relations qui unissaient les deux manuscrits? Notre recherche permet d'établir le processus suivant qui concerne surtout les pages 1-140 du manuscrit de Webster. Tout d'abord Vinson corrige une partie de brouillon de Webster (Vinson ne corrige pas toutes 
ses fautes, il ne corrige pas souvent les sifflantes, il corrige plutôt ce qui est important pour la compréhension du texte), il préfère recopier le reste. Ensuite, Webster réécrit une partie du manuscrit de Vinson (dans le premier cahier il maîtrise beaucoup mieux la langue basque, il corrige même les fautes que Vinson n'a pas corrigées, la ponctuation y est ajoutée).

\section{$\mathrm{W} 1>\mathrm{V}>\mathrm{W} 2>$ Vinson et une autre personne ont vu le texte de Webster}

Pour les contes qui n'ont pas de deuxième variante basque dans le manuscrit de Webster, on restitue le processus suivant :

W0 (brouillon qu'on n'a pas) > W1 (pour certains contes après avoir consulté la lettre de Vinson) $>$ V

Passons aux commentaires. Dans le manuscrit de Webster, il y a plusieurs contes ayant deux variantes en basque qui correspondent à une variante chez Vinson. Parmi les deux variantes la première est secondaire, et la deuxième ressemble plutôt à un brouillon (la variante du premier cahier tient conte des corrections faites par Vinson dans le deuxième cahier et cahiers suivants): Dans le troisième cahier Webster fait moins de fautes, et c'est une raison pour laquelle on peut supposer que chronologiquement le deuxième cahier de Webster était le premier. Donc, il y a eu a eu plusieurs étapes de coopération de deux folkloristes. Il est possible de parler de deux sous-corpus de Webster : avant la correction de Vinson et après la correction de Vinson.

Comment Vinson recopie-t-il le manuscrit de Webster? Vinson recopie tous les contes du manuscrit de Webster sans exception (cependant il ne recopie pas les quelques devinettes, ni le conte écrit par la fille de M. Webster (qui pouvaient également être ajoutés au manuscrit plus tard). Après avoir corrigé quelques textes de Webster, Vinson a finalement décidé de ne pas continuer les corrections. Il les a recopiés directement du manuscrit de Webster. Tout en recopiant le premier cahier de Webster, (les contes I-XIX chez Vinson, pages 193-234 chez Webster) après le conte Sorginak Akhelarrian (pages 235-236), Vinson recopie deux contes qu'on ne trouve pas dans le manuscrit de Webster (XX, XXI), Apheza eta andrea et Emazteak. Le conte XXII correspond aux pages 248-250 du manuscrit de Webster. On ne retrouve non plus le conte 88 dans le recueil de Webster.

Dans les deux manuscrits on voit des remarques au crayon bleu qui pourrait être le même ([W454v, 494r, 519v, 522v, 523v, 525v, 526v ; V163]). Dans le premier cahier de Webster [W15], on voit des corrections au crayon, il s'agit d'une écriture qui ressemble beaucoup à celle de Vinson (peut-être que les deux folkloristes se sont échangés des cahiers à plusieurs reprises).

Il existe des cas où Vinson restitue des formules traditionnelles du manuscrit de son collègue, cf. « Orduan han ninzen » [W427r] où Vinson ajoute « eta orai hemen » [V131]. Vinson corrige l'orthographe de Webster. Celle de Vinson est beaucoup plus régulière. Il corrige également des fautes d'incompréhension (« diruak ematen du pobre eri » [W192] vs. « diruak ematen tu pobrieri » [V272]). Une fois, le folkloriste supprime une répétition de Webster : « behar duzu gan lehembizi behar duzu gan aphez baten biła » [W586r-587r] vs. «Behar duzu gan lehembizi aphez baten billa » [V237]. Parfois, Vinson change l'ordre des mots : « Bazuan kargatua erreka batean Nafarras » [W463] vs. « Bazuan erreka bateat Nafarraz kargatua » [V153] (très probablement, il ne le fait pas exprès). Vinson change rarement la grammaire : «ematen diote » [W300r] vs. « ematen dute » [V270].

En réécrivant les contes, il est assez facile de sauter quelques mots. Cela veut dire, que si on compare deux textes, dont un a été réécrit, le texte original doit être plus complet et doit 
contenir les mots qui sont absents dans la version réécrite.

Vinson saute des phrases de Webster, ce qui nous aide à restituer la chronologie de travail avec les deux manuscrits : Ainsi, « pensa neskatcho gaso haren penak eta doloriak bera ala gamber duela eraten dio bere burua bestitzen da » [W253] est une phrase qui est absente chez Vinson, cf. [V72].

Nous citerons également un autre exemple :

« Badohazi bañian gizon hekiek pena zuten neschatcha hura hilzea arras poułitazen eta erraten zioten inozenta zela » [W570r].

« Badohazi bañan pullita zen eta erraten zioten inozenta zela» [V225].

D'autres exemples des mots que Vinson saute se trouvent sur les pages [W487r, 506r, 528r, 529r, 541r, 563r, 567r].

Dans le premier cahier de Webster on voit que le folkloriste anglais saute quelques mots du texte de Vinson, en le réécrivant (compte tenu de son respect pour son ami savant, il est très peu probable que Webster l'ait fait volontairement):

[W3] «Eta egun batez atsematen du neskatchak eztu nahi arras izitu baitzuen ».

[V1] «Eta egun batez atsematen du neskatcha pulit bat, arras gustatu zen, bainan neskatchak eztu nahi arras izitu baitzuen ».

Il est évident que Vinson n'a pas inventé les mots manquants, puisque on les trouve dans la partie du manuscrit de Webster qui contient le plus de fautes, donc qui est la version précédente:

[W193] « eta egun bates atchimatendu neskatcha pouli bat arras gustatucen bainan neskatcha estu nahi is/z/ito baizuen ».

On trouve des exemples semblables dans plusieurs contes. Dans le conte Acheria, Webster omet les mots qui se situent entre le même mot « egia » :

[W9] « orai erditan gaituk, errak bigarren egia; eztuk sekulan artoa ogia bezin ona »

[V3] « orai erditan gaituk errak bigarren egia; erraten dio gizonak oy! cer arto ona! iduri du oguia! bainan eztuk egia eztuk sekulan artoa ogia bezin ona »

[W194] « orai erditas gaito erak bigarren eguia eraten dio guizonac oyhau harto idurid/t/u oguia bainan eztuk eguia eztuk sekula hartua ogua besin ona ».

Si on compare les pages [W47] et [V9], on verra que Webster a sauté quelques mots en recopiant le texte de Vinson. Un autre exemple se présente, dans son premier cahier, cf. [W67]. Après avoir copié la page [V18], Webster, au lieu de copier [V19], commence à copier [V17] dès le début : " erraten dio : orai hartzak zigor bat eta emok, oihanian sartzean, harekin sarteko bat lurrari [...]» [W67-69]. Après avoir recopié quelques lignes, Webster se rend compte qu'il s'est trompé et rature les lignes. En réécrivant le texte sur la page 213, Webster en omet un morceau de quelques lignes et puis, le copie sur la partie verso de la page précédente. De cette façon, on peut être sûr que Webster réécrit le texte de Vinson.

Nous pouvons également prouver pour quelques contes que Webster a recopié le manuscrit de Vinson, en analysant les fautes de celui-ci. Si on compare le texte dans les pages [W37] et [V9], on remarquera le texte " gauza herak», au lieu de " gauza berak » dans le manuscrit de Webster. Normalement, Webster fait la différence entre b et h, tandis que dans le manuscrit de Vinson de fois b ressemble à h.

En réécrivant le manuscrit de Vinson, Webster suit l'ordre de ses contes, tout en copiant son texte très attentivement. Pour comparer le texte de Webster et le texte de Vinson plus en détail, on va citer le texte du même conte, Tartalo, où on remarque que Vinson a copié le texte 
de Webster.

[W3] La première version de Webster — chronologiquement la deuxième

Tartarua. B. L. 4.

J. Vinson, Le folklore du Pays Basque, p. 46. <une ligne ajoutée plus tard>

Behin batez, bazen Errege seme bat, punimendu batez bezala mustro bat gelditua; eta etzitaken yende bilaka escondu arte. Eta egun batez atsematen neskatchak eztu nahi arras izitu baitzuen. Eta Tartato harek nahi zion eman errestun bat, eta neskatchak etzuen nahi hartu; bainan yaun gazte batekin egortzen dio, eta errestunak, erhian sartu orduko, erraten zuen: «hi hor eta ni hemen.» bethi oyhuka eta Tartarua bethi segitzen da, eta neskatchak izitzen baitzen, erhia pikatzen du bere erhestunarekin eta botatzen du ur handi baterat, eta han itotzen da Tartarua.

\section{Estefanella Hiragaray}

[W193] La deuxième version de Webster - chronologiquement la première <nous ne transcrivons que le texte de Webster, sans corrections de Vinson qui y ont été ajoutées plus $\operatorname{tard}>$

Tartarua

Behin bates basen Errege seme bat punimendu bates besala mustro bat gelditua eta etchitaken yendes bilaka esconduarte eta egun bates atchimatendu neskatcha pouli bat arras gustatucen bainan neskatcha estu nahi is/z/ito baizuen Eta Tartato harek nahi z/s/ion eman errestumbat eta neskatcha es dai nahi harto bainan juangazte bateken egortsan dio eta errestunac errian sartu orduko eeraten du ihor eta ni emen beti hoyuka eta Tartarua beti segitzenda eta neskatcha izizten baitzen heria pikatzendu bere erestunarekin eta botatzendu ou handi baterat eta han itotzen da Tartarua.

[V1] La version de Vinson

I. Tartarua. W. p-4

Behin batez, bazen Errege seme bat, punimendu batez bezala mustro bat gelditua; eta etzitaken yende bilaka ezcondu arte. Eta egun batez atsematen du neskatcha pulit bat, arras gustatu zen, bainan neskatchak eztu nahi arras izitu baitzuen. Eta Tartato harek nahi zion eman errestun bat, eta neskatchak etzuen nahi hartu; bainan yaun gazte batekin egortzen dio, eta errestunak, erhian sartu orduko, erraten zuen: «hi hor eta ni hemen», bethi oyhuka eta Tartarua bethi segitzen da, eta neskatchak izitzen baitzen, erhia pikatzen du bere errestunarekin eta botatzen du ur handi baterat, eta han itotzen da Tartarua.

\section{Estefana Hirigaray}

La comparaison de la (chronologiquement) deuxième version de Webster et de la version de Vinson [W3] et [V1]

\section{Tartarua.}

Behin batez, bazen Errege seme bat, punimendu batez bezala mustro bat gelditua; eta etzitaken yende bilaka eszzcondu arte. Eta egun batez atsematen du neskatcha pulit bat, arras gustatu zen, bainan neskatchak eztu nahi arras izitu baitzuen. Eta Tartato harek nahi zion eman errestun bat, eta neskatchak etzuen nahi hartu; bainan yaun gazte batekin egortzen dio, eta errestunak, erhian sartu orduko, erraten zuen: «hi hor eta ni hemen_», bethi oyhuka eta Tartarua 
bethi segitzen da, eta neskatchak izitzen baitzen, erhia pikatzen du bere errhestunarekin eta botatzen du ur handi baterat, eta han itotzen da Tartarua.

\section{Estefanella Hirigaray}

La comparaison de la (chronologiquement) première version de Webster et de la version de Vinson [W193] et [V1] ${ }^{10}$ Tartarua

Behin bates $z$ baszen Errege seme bat punimendu bates $z$ beșzala mustro bat gelditua eta

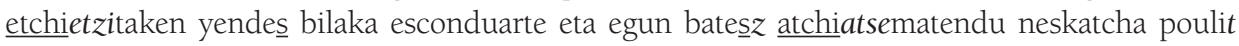
bat arras gustatuctzen bainan neskatchak estu nahi is/z/ito baitzuen Eta Tartato harek nahi z/s/ ion eman errestumbat eta neskatchak es dai etzuen nahi hartou bainan juaaungazte bateken egortsanzen dio eta errestunac errian sartu orduko eerraten du zuen ihor hi hor eta ni emen beti hoyuka oyhuka eta Tartarua beti segitzenda eta neskatchak izizttzen baitzen heria erhia pikatzendu bere errestunarekin eta botatzendu ou ur handi baterat eta han itotzen da Tartarua.

\section{Estefana Hirigaray}

Vinson ne laisse ses remarques dans le manuscrit de Webster que très rarement : sur la page [W368], on en voit une: "This tale is in a very bad basque ; it seems to me have been translated from french viz. from a french book J. V. » (pourtant, Cuzacq affirme que la même légende est extrêmement connue : "s sur la lancée des écrivains des XVII et XVIII ${ }^{\text {ème }}$ siècles, notre légende, restée populaire, abonde encore largement au XIX ${ }^{\text {ème }}$ siècle, et d'abord en sa première moitié » [Cuzacq 1975 : 193]).

Il nous reste encore une question concernant les relations entre les deux manuscrits : pourquoi Webster a réécrit le texte basque de Vinson qui était de très bonne qualité ? Probablement, Vinson voulait le garder pour lui, et Webster désirait le déposer dans un endroit où il pourrait être publié.

\section{La lettre de Vinson à Webster et ses conséquences}

La relation entre les deux folkloristes s'exprime dans une lettre que Vinson écrit à Webster en anglais et en français sur une simple feuille de papier (elle se trouvait probablement dans les cahiers que Vinson a renvoyés à Webster par courrier). Plus précisément, il ne s'agit pas d'une lettre, mais de certaines remarques concernant le manuscrit, puisque on n'y trouve pas de salutation traditionnelle. Maintenant cette lettre est collée dans le manuscrit à la page [W618v], et on peut supposer que Vinson l'a renvoyée à Webster en même temps que les cahiers.

Nous citerons cette lettre où il s'agit des fautes typiques du prêtre anglais ${ }^{11}$.

recto: «I rend you back, dear sir, your books and cahiers, with a vol. from M. Nogaret \& 2 $\mathrm{n}$ of a newspaper (with an article of mine; one for Sayce ${ }^{12}$ to whom I will write soon).

10. Beaucoup de corrections sont déjà ajoutées au texte de Webster par Vinson.

11. Il faut ajouter que Vinson a commencé à apprendre la langue basque par lui-même en 1866, après avoir pris « possession de l'emploi de garde général des forêt à Bayonne ». Il devient sous-inspecteur à Bagnères de Bigorre en 1878 et revient à Paris, sa ville natale, en 1879 [Lacombe 1927 : 61]

12. A cause de l'écriture difficile à déchiffrer de Webster, nous ne savons pas, si nous avons transcrit le nom correctement ; il s'agit probablement de M. Sayce que Vinson mentionne dans son annexe du livre Basque Legends [Webster $1879: 21$ ]. 
Pray read me other cahiers and academies if them be any thing. Observations in your tales:

$1^{\circ}$ don't confound herri country \& hiri city naiz I am \& nahiz although beriak (bereak) their \& berriak new

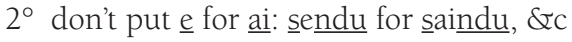
nor put h where it is unpronounced. So you must write seurki not sehurki (correct segurki)

$3^{\circ}$ write always choilki (not seulki), heian or heyan, eyan (not Iyan) "whether»,

$4^{\circ}$ vérifiez si l'on dit haren handia, harembertze, hartaz bertzerik, etc. Il me semble qu'on dit plutôt hain handia, hainbertze, hartaz baizik

$5^{\circ}$ don't write zigoen baligoa, but zioen, balioa, or ziuen, baliua or wighter ziyoen, baliyoa (y not g)

$6^{\circ}$ your translations are sometimes not perfect, viz.» verso: «you put on french the first person when the bask was the 3. Erran zuen ethorriko dela "Il a dit qu'il viendra"13, not "il a dit: je viendrais".

I don't know whom I'll be you. We will make our classification of tales at out first meeting.

A good new [sic]. My work on bask, translated from hungarian and annotated, is under the press. I corrected yesterday the 1 proof.

Yours' truely,

<signature>»

La date de publication de l'œuvre dont Vinson parle serait très importante pour savoir en quelle année la lettre a été écrite. Il s'agit très probablement de l'Essay sur la langue basque de François (Ferencz) Ribáry publié à Paris en 1877 (Selon Julio de Urquijo, le travail a été traduit en 1876) - c'est la seule traduction du hongrois de Vinson que nous avons pu trouver ${ }^{14}$. Comme Vinson date son manuscrit de 1876-1878, la lettre a été écrite lorsque la plupart des contes n'avaient pas été encore copiés.

En analysant la lettre, on voit que Vinson a déjà copié le texte de plusieurs des derniers cahiers de Webster (la partie où l'on ne voit presque pas de ratures), puisqu'on trouve le mot « seulki » dans son manuscrit sur les pages [W345r, 410r, 428r, 441r et 514r] (chaque fois,

13. Webster écrit « avait » au-dessous de « a » et « viendrait» au-dessous de « viendra ». Il y a encore quel ques mots écrits par Webster sur le côté recto de la lettre.

14. Lhistoire de cette traduction est intéressante en soi : Vinson a appris le hongrois pour le traduire : « En 1876, j'ai voulu traduire en français l'Essay sur la langue basque écrit en hongrois par M. Fr. Ribáry, professeur à l'Université de Buda-Pest. Que-je fait pour y arriver en peu de temps ? Je me suis procuré un petit dictionnaire magyare-français et une grammaire magyare quelconque ; j'ai commencé par lire cette grammaire, la plume à la main, et j'en ai résumé la partie essentielle (déclinaisons et conjugaisons) en quelques tableaux. Puis, je me suis attaqué au texte. Le commencement a été pénible : le sens général se détachait difficilement des mots rapprochés; mais dès la dixième page tout était plus claire ; à la cinquantième le travail devenait facile, à la fin je traduisait presque à livre ouvert» [Vinson 1899 : vii]. 
Vinson dans son manuscrit le réécrit comme « choilki ») et « sendu » au lieu de «saindu » sur les pages [W253, 289r, 293r, 294r, 397r, 408r, 426r]. Cependant, sur les pages suivantes du manuscrit de Webster [W550r, 571r, 590r, 597r, 610r et 611r], celui-ci tient compte de la remarque de Vinson et écrit régulièrement « choilki ».

Pourtant, on peut être sûr qu'il ne copie pas plusieurs contes réécrits par Vinson (HerenSugea [W141-161, Errua [W163-177], ainsi que la plupart des contes du troisième cahier), puisque, contrairement au premier cahier, il y a une différence importante entre les deux versions des folkloristes : "Harat gau direnian sah/g/ar lore guziak jaun haren ganeat zoazin behorra ere arizen aizeka eta Prinzeak etzuen izatu lore bat choilki. » [W610r] vs. « Haat gau direnian saar lore guziak yaun haren gañeat zoazin Behorra ere hari zen aizeka eta printzeak etzuen izatu lore bat choilki » [V255].

Grâce aux contes basques Webster apprend la langue et l'orthographe basque. Ainsi, si dans à partir de la page [W193] jusqu'à la page [W497r], il écrit le verbe «erran » 'dire' avec un r, a partir de la page [W498r] il commence à l'écrire correctement, avec deux r.

D'autres fautes d'orthographe sont plus difficiles à corriger. En ce qui concerne la remarque sur les deux mots « beriak » 'leurs, les mêmes' et « berriak » 'nouveau, les nouvelles', Webster les confond dans tout le texte du manuscrit, sauf le premier cahier qui a été copié du manuscrit de Vinson. Cela peut être expliqué par le fait que la différence sémantique entre ces deux mots demande plus de compétences linguistiques que la prise en compte de la remarque précédente.

En conclusion, on voudrait ajouter qu'il serait souhaitable de publier le texte basque, français et anglais du manuscrit de Webster. Il serait également nécessaire de publier le facsimilé de deux manuscrits qui serait utile pour des recherches textologiques (cf. l'avis de Lebrave : "Le fac-similé reste indispensable. Aucune des formes d'édition envisageables n'appréhende la substance graphique du manuscrit dans sa totalité et ne peut donc mettre fin à un commentaire de celle-ci par nature indispensable» [Lebrave 2006 : 15] .

\section{La traduction et les compétences linguistiques de Webster}

Il est très étonnant qu'un des meilleurs recueils des contes basques ait été fait par une personne qui connaissait peu la langue et qui l'a apprise dans une grande mesure en recopiant les contes (les enfants de Webster savaient parler basque [Urquizu 2000 : 3]). A présent, il convient d'étudier les compétences linguistiques de Webster, ainsi qu’analyser la fidélité au texte basque.

\section{Les modifications du texte}

Dans un article consacré aux contes de Cerquand, Webster déclare ses principes de la fidélité absolue au texte : en transcrivant les contes, « il faut être aussi exact et aussi rigoureux à les écrire "impsissima verba", que ce serait un helléniste avec un vieux palimpseste grec » [Webster 1875: 174]. Le folkloriste n'essaie pas de corriger les éléments innovateurs qu'introduisent les locuteurs (« un jour pendant qu'il était à lire » [W269]). En revanche, Webster remarque les défauts des contes, en les commentant dans les notes de bas de page, par exemple, «Here the narrator evidently forgot to tell about the child's being exposed, and the gardener finding it, as appears by the sequel » [Webster 1879: 177].

Les traductions de Webster sont très littérales par rapport aux contes de Cerquand, même lorsqu'il s'agit des contes merveilleux, que les folkloristes modifient le plus souvent. Webster se montre très réservé à l'égard de sa traduction : "Our translations are literal to badness; the only liberty we have taken is in softening down the exceeding directness and grossness 
of some portions » [Webster 1879 : viii]. Lanalyse du manuscrit et de la traduction du livre, permet d'établir que dans ce passage, il s'agit d'euphémismes. Comme ils sont peu nombreux, on va en citer quelques-uns :

«bere buruko minia sendatua izen dubela lehenbiziko atsemanen duen asto-pisaekin buruba garbitu eta » [W331r-332r] cf. « pour vous guérir le mal de votre tête le premier âne que vous trouverez et qu'il invitera vous vous laverez avec cela [W330v-331v].

«Galdetzen dio Tartaruak : Zer marka uten haren cherriak? Eraten dio erruak: Nere hik buztan azpian ziło bat zulobi ematen dire» [W23] (Tartaro lui demande : Quelle marque ont ses porcs? Le fou lui dit: Les miens ont un trou sous la queue, on dirait une caverne), cf. "The Tartaro asked the madman what mark his pigs had. The madman says to him, "Mine have some of them one mark, some of them two marks" » [Webster 1879: 9]. Cet euphémisme est également présent dans la traduction de Bourguète : " Le Tartaro demanda au fou quelle marque portaient les cochons. Le fou lui dit : «Certains des miens ont une marque, d'autres deux marques » [Webster 2005 : 18].

Dans le conte Ezkabi-Fidel, on trouve des lignes suivantes: « Oua goiti eta ekharrak kasola bat eta nik pisa ite utenian garbituko haiz eskubak eta buruba » [W560r] — « Go and fetch a saucepan, and when I shall have filled it with water, you will wash your hands and your head » [Webster 1879 : 112] (on ne comprend pas comment la jument doit remplir la casserole de l'eau).

On trouve encore quelques euphémismes dans le conte Andre usoa eta bere orratzea ( $\mathrm{La}$ dame Pigeon et sa peigne) :

« Andre gazteak erraten dio arratchean Aitak erran zituen garraño hura nere aita izaen da yoko uzu iñdar guziez zure makil izigarri harrekin suh/d/ur/narines/ frinzetan pisa espakatuko zaiyo eta garritua izaen da yoko uzu bularetan zure iñi[ ]alekin hari ere pisa espakatuko zaiyo eta benzutua izaen da Ni azkenik ethorriko naiz iñien duzu alegietan ni yo eta yoko uzu zure makiłaekin lurra nik ere iñien dut pisa eta garraituba izaen naiz » [W583v].

« The young lady says to him in the evening: "The horse which my father has spoken to you about will be himself. You will strike him with all your might with your terrible club on the nose, and he will yield and be conquered. The first filly will be my eldest sister. You will strike her on the chest with all your force, and she also will yield and will be conquered. I shall come the last. You will make a show of beating me too, and you will hit the ground with your stick, and I too will yield, and I shall be conquered » [Webster 1879 : 124].

D'autres petites adaptations concernent les mots qui sont difficiles à comprendre dans l'original : « Agur, agur, otso yauna baldin egarri bahaiz badiat Nafarrona edateko» [W465r] vs. « Good morning, good morning, Mr. Wolf; in case you should be thirsty, I have some excellent Malaga to drink» [Webster 1879: 45].

Bien que la traduction de Webster soit presque littérale, parfois il change légèrement le sens des mots: « Eraten diote Iyan lay/ih/orra emaen zioten ikhusten zuten yauregui hartan » [W286r] — « They ask her if shelter would be given them in the house which they see there » [Webster 1879 : 203].

Plusieurs notes servent à spécifier les pronoms, cf. « eta horekin batean ematen dio charsta bat » [W151], « Et en même temps il (le pasteur) lui donne un petit coup » [W382v] vs. « And at the same time the shepherd gives him a little blow» [W148]. Ces changements peut-être sont dus aux corrections de Julien Vinson qui suivait le texte basque. Dans les traductions, les formules ne sont pas traduites littéralement, cf. le basque «Comme bien souvent dans ce 
monde » dans la traduction française et « Like many others in the world » dans la traduction anglaise (une formule qu'on trouve dans beacoup des contes de Webster). Parfois, Webster remplace le pronom par son antécédent pour que le texte soit plus claire, cf. " elles font croire à la mère » [W348] vs. " her sisters made the queen believe » [Webster 1879: 178]), mais le sens reste le même.

Cependant, malgré ces petits changements que nous avons décrits, la traduction des textes est assez fidèle, ce qui est plutôt rare chez les folkloristes basques. Un chercheur sur les contes de Mme Aulnoy, N. Jasmin remarque : «L'une des tendances dominantes des contes est en effet l'euphémisation de la matière folklorique » [Jasmin 2008 : 27]. Si Mme Aulnoy supprime les détails scabreux et trop réalistes, la plupart des adaptateurs modernes ne suppriment que les premiers, en ajoutant aux contes les éléments de la couleur locale.

\section{Les compétences de Webster en basque}

Dans les études précédentes, on a remarqué que Webster ne maîtrisait pas parfaitement la langue basque : «M. Webster lui-même, bien qu'il fût arrivé à comprendre la langue, et à en posséder une haute connaissance scientifique, défendit toujours d'être jamais parvenu à s'exprimer couramment en « eskuara » [Veyrin 1955: 311]. Son ami Vinson écrit en 1921 dans une lettre publiée dans Gure Herria : «Webster s'est occupé des Basques, de leurs mœurs et de leur histoire, mais il n'en savait pas la langue» [Vinson 1921 : 682]. D'autres chercheurs, y compris, Charles Videgain, y voient un défaut, cf. « Le texte basque de Webster n'est pas d'une fidélité totale, non seulement sans valeur phonétique, mais aussi parce que Webster savait mal le basque » [Irigarai, Videgain 1974 : 463].

Les chercheurs qui ont travaillé sur le manuscrit de Webster remarquent que le texte original du manuscrit contient beaucoup de fautes qui sont dues aux informants de Webster et à Webster même ( « cuentos de Webster [... ] están escritos [... ] con bastantes faltas y omisiones, atribuibles a Mme Bellevue, más bien que al o a la comunicante de turno. Tampoco Webster, que se confió a aquella señora, parece que dominaba a fondo el euskera [...] » [Irigarai, Videgain 1974: 451]).

En analysant la transcription, on peut conclure qu'au début de son travail il ne parlait pas basque assez bien pour traduire les contes directement en anglais. La segmentation erronée de Webster prouve que ses compétences linguistiques n'étaient pas parfaites. Ici, nous citerons plusieurs exemples où Webster ne comprend pas le texte qu'il note : "gizen duazen » (gizendua zen) [W603r], « guizon gahi » (gizongai) [W602r], « bortchea tuada » (bortchatua da) [W215r], « aitu kotuela » (aituko tuela) [215v], «obligatu bada» (obligatua da) [W212], « basa uste (bazauzte)» [W196], « arribakotcha hari » (harri bakotchari) [W254r], « arribiła katuak » (harri bilakatuak) [W254r], « pobre eri » pobrieri [W292r], « indu zube » (in duzube) [W293r], « sendu ba en » (saindubaen) [W293r], « basa kizkian » bazakizkien [W299r], « laster kadoana » (lasterka doana) [W303r]. Parfois c'est lié aux problèmes de la compréhencion : « galde hasten » (galdaazten) [W211], « bere alaba (berehala) [W261]. La plupart de ces exemples concernent les premières versions des textes, avant la correction de Vinson.

On peut beaucoup apprendre à partir des gloses, c'est-à-dire, des traductions qui Webster donne au-dessus et au-dessous des mots. Les premiers mots qu'il note témoignent de son début des études en basque (les mots très courants qui se repèrent en plus dans le grand nombre des contes), cf. oihan/forêt/, anaya/le frère/ [W196], atsimatendu/elle a trouvé/ [W217], senarra/ 
l'epoux/ [W261]. Exceptionnellement, Webster ajoute des remarques en espagnol, au crayon, cf. zortzi bat/unos/ [W511r]. Dans le texte basque de Webster on voit des corrections au niveau du lexique : doctor/miriko/ [W363r]. Il est possible que Webster ait demandé à ses interlocuteurs de répéter le texte plusieurs fois, ou c'était un cas d'autocorrection de la variante moins basque vers la variante plus basque.

De l'autre côté, dans le livre Basque Legends, Webster donne une opinion qui n'est pas complètement acceptable, mais elle prouve que le folkloriste est capable d'analyser le texte basque: "The blunder is confounding "dessus", over, and "dessous", under. This shows that the tale is originally French, or, at least, the witch's part of it; for this punning mistake could not be made in Basque. The two words are not in the least similar in sound. "Gaiñetik" and "azpetik" are the words here used» [Webster 1879: 70].

\section{Les compétences de Webster en français}

Dans la traduction française du manuscrit de Webster, on peut trouver des fautes grammaticales, telles que l'emploi de l'indicatif au lieu du subjonctif : "Que celle qui a fait cette galette vient [sic] devant moi » [W380] ; " pour qu'elle ne meurt [sic] pas de faim » [W349]. Les fautes d'articles sont assez rares : « il s'arrête dans un [sic] auberge [W412v], « dans le [sic] montagne » [W339v]. Très souvent on peut remarquer les fautes concernant l'accord du verbe et du genre dans les syntagmes nominaux, surtout lorsqu'il s'agit des homophones : « on les tuaient » $[\mathrm{W} 412 \mathrm{v}]$; « telle [sic] roi » $[\mathrm{W} 415 \mathrm{v}]$; « Ils reste [sic] toujours attendants » [W417v] ; « ils [sic] trouve une vieille vieille dame » [W381] ; « Il avait entendu leur [sic] paroles » [W347], « cet village » [W360v]), mais on rencontre aussi des cas où la prononciation est différente : «l'héritier de Belzunce etait pensive » [W65v]. Les fautes d'orthographe concernant les homophones sont aussi courants : "Cette pauvre mer aurait voulu voir son fils prend une femme » [W397v]. Cependant, en général, il parle et écrit français assez bien, ce qui est normal pour un pasteur qui s'installe en France et fait publier beaucoup d'œuvres en français.

Dans la version française Webster omet souvent les signes de la ponctuation, parfois son orthographe est incertaine (nous avons déjà cité quelques fautes du manuscrit). Ainsi, il oublie parfois de mettre les accents (ainées [W314], faché [W422v], diner [W423v]). De temps en temps il a des problèmes avec l'accord des participes (« elle s'était habille avec la belle robe qu'elle avait porté » [W421v], Quand elle est arrivé [W379]). A titre d'exemple nous citerons un extrait en français :

«Et elle lui reponde [sic] oui mon père si vous n'avez que ca [sic], ne vous chagrinez pas j'irais volontiers Quand les deux ainées ont appris cela elles se sont rejouies Elles sont très [sic] parce qu'elles pensent que le monstre leur [sic] mangera » [W314-315]

En revanche, l'orthographe et la ponctuation de sa version anglaise sont très soignées. Parfois Webster marque dans le texte français la traduction littérale du basque. Ainsi, dans la traduction d'un des contes d'Heren-Suge Webster donne la traduction « froid » ( hotza ») au-dessus du texte « et il tombe raide mort » [W382r].

Il est évident que le texte français a été dicté à Webster à cause de l'orthographe incertaine du folkloriste (e. g. « dessuos » [W380], « flaçon » [W335v] et d'autres). Parfois on remarque certaines fautes qui sont dues au caractère provisoire de la traduction (« voudrais-tu aller chez dans ta maison » [W465r]). En comparant le texte basque et le texte français, on peut remarquer l'influence de la langue basque dans la construction suivante : " Hiru hauride » 
[W214] vs. "Les trois frères et sœurs » [W349] et " The two brothers and their sister » [Webster 1879: 179]. Parfois, la traduction française reflète la structure de l'énoncé basque: « heldu zaio karosa eder bat » [W105] « il lui vient une belle voiture » [W379] ; l'inversion selon le modèle basque « Egun batez erraten diote [sic] neskatcha harek » [W107] vs. "Un jour leur dit cette jeune fille»[W379]. Cependant, dans des cas pareils, on ne peut pas affirmer qu'il s'agisse des compétences linguistiques de Webster: il s'agit probablement de la traduction de Mme Bellevue.

Dans la traduction française de Webster on remarque des cas de code-switching : « Le maitre de l'auberge va tout de suite dans la maison du roi et on lui dit qu'il leur en faut que oui Et Eskabi goes there [sic]. Comme il etait tard [...]» [W332v]). On peut supposer que le folkloriste lors qu'il écrivait la traduction française sous la dictée de Mme Bellevue a inséré les mots anglais automatiquement.

Même maintenant les éditeurs et traducteurs parfois ne comprennent pas complètement le processus de la traduction des contes. Ainsi, Burguète écrit dans son avant-propos : « La traduction qu'il fit de ces légendes basques en anglais est aussi littérale que possible...» [Webster 2005 : 13-14], sans mentionner que le texte basque a été traduit en français et puis en anglais. Cependant, on peut affirmer que le folkloriste anglais a traduit le texte dans sa langue maternelle, en utilisant la version de Mme de Bellevue, et pas celle de Vinson qui a été publiée quelques années plus tard, dans le Folklore du Pays Basque. Pour le prouver, il suffit de comparer les versions du conte Malbrouk : «sa femme était sur le point d'accoucher » [W464v], «His wife was on the point of being confined» [Webster 1879 : 77] « La femme redevint enceinte » [Vinson 1883: 80].

Ayant analysé toutes les versions du manuscrit de Webster, ainsi que la réécriture de Vinson, on voit des progrès considérables de la compétence linguistique de Webster.

\section{Le manuscrit et le livre Basque Legends publié en anglais}

\section{Remarques générales}

Bien que les textes de Webster soient étudiés, les folkloristes se rendaient compte qu'une partie importante du manuscrit restait inédite. Les éditions anciennes des contes basques de Webster sont peu nombreuses. Comme nous l'avons déjà dit, le recueil de Webster a été imprimé pour la première fois en anglais, en 1877, et la deuxième édition a paru en 1879 (elle comprend également un chapitre sur la poésie basque). Quelques contes de Webster ont été publiés par Julien Vinson en français dans ses recueils Les Basques et le Pays Basque (Paris, 1882) et Le Folklore du Pays Basque (Paris, 1883). Selon P. Urquizu, plusieurs contes de Webster ont étépubliés par Sébillot dans le recueil Contes des Provinces de France [Urquizu $2000: 7]$.

La première édition des contes de Webster sous forme d'un livre a paru en anglais. Ce fait est probablement dû à la volonté de Webster de publier les contes dans sa langue maternelle et d'avoir la possibilité de travailler avec le texte ainsi qu'au désir que ses compatriotes puissent accéder au texte. Les contes ont été édités peu de temps après qu'ils aient été recueillis : la plupart des contes ont été recueillis en 1874 et 1875, et la première édition est parue en anglais en 1877. Webster a dû tenir compte du travail de Vinson avant la publication de son livre, puisque le texte de Vinson date des années 1876-1878.

Dans le recueil de contes déjà publié, Webster classe les contes en fonction des sujets, une 
classification complètement absente dans le manuscrit. En parlant des relations entre les deux folkloristes, il faut ajouter que probablement, la classification de Webster, a été influencée par Vinson, puisque celui-ci en parle dans sa lettre. On peut supposer qu'une rencontre au moins a eu lieu au Pays Basque, puisque Vinson, dans son annexe An essay on the Basque language indique la date et le lieu: Bayonne, August 28, 1876.

Une moitié des contes en basque n'a pas été publiée en français et en anglais (Il est à souligner qu'il faudrait les publier en anglais, pour que le lecteur anglophone puisse accéder à ces textes, comme il semble assez incroyable que les textes du fameux folkloriste n'aient pas été publiés dans sa langue maternelle).

\section{Les commentaires}

Dans le texte imprimé on remarque aussi les notes de bas de page qui parfois proviennent des notes de bas de page et d'autres remarques trouvés dans le manuscrit de Webster. Ce dernier y ajoute des commentaires ethnographiques et culturels, tandis que dans le manuscrit on voit plutôt des commentaires concernant le procédé de la collecte. On donnera un exemple d'un commentaire du livre: "We have other tales about priests, all in the same spirit as this. The Basques are a deeply religious people, and are generally on the best terms with the clergy; but they will not be dominated by them. Any attempt at undue interference in their national games or customs is sure to be resented; of this we have known several instances some rather amusing ones ». Bien que Webster indique parfois l'origine des contes et qu'il donne les parallèles dans d'autres recueils, ces observations ne sont point systématiques et exigent un travail plus profond.

En analysant les textes des contes, Webster fait non seulement des commentaires comparant le folklore basque à celui d'autres peuples européens, mais aussi aux premiers contes recueillis au Pays Basque par Cerquand. Vers l'époque où le recueil de Webster a été publié pour la première fois (en 1877), le folkloriste anglais a eu accès à deux tomes du Bulletin où Cerquand avait publié ses contes, cf. : «Of the latter part of our first story we have heard many variations. In one given by M. Cerquand, p. 29, the fox is represented by Basa-Jauna; in a version from Baigorry, by the Tartaro ${ }^{15} » ;$ « None of these legends have been published or even noticed till within the last two years, when M. d'Abbadie read the legend of the Tartaro, before the Société des Sciences et des Arts de Bayonne, and M. Cerquand his "Légendes et Récits Populaires du Pays Basque," before the sister society at Pau» [Webster 1879 : viii]. En revanche, Cerquand a pu utiliser les contes de Webster dans la 3 et $4^{\text {èmeème }}$ parties lors de la publication des contes basques dans le Bulletin de la Société des Sciences, Lettres et Arts de Pau, ce qu'on voit dans ses commentaires : «M. Antoine d'Abbadie a donné du conte [de Petit Poucet] une version d'Esquile, reproduite, ainsi qu'une version très-abrégée d'Ahetze, dans les Basque Legends du R. Wentworth Webster, p. 4 et 5. London, Griffith et Farran, 1877 » [Cerquand $1877: 457$ ], « Le rév. W. Webster a donné ce conte : Basque Legends, p. 58 »[Cerquans 1883 : 144] etc.

15. Webster fait une reférence aux «Légendes et Récits Populaires du Pays Basque," par M. Cerquand. PartI., Pau, 1875, and Part II., p. 28, Pau, 1876». 
Dans le livre de Basque legends les données concernant les informants ne sont pas cohérentes, bien que Webster ait pu éviter ce défaut, ayant souvent le nom et le prénom du conteur dans son manuscrit. Parfois, il ne donne pas du tout la source des contes. Dans d'autres cas, il marque le prénom sans le nom. Il lui arrive, cependant, de fournir des commentaires plus élargis : " PIERRE BERTRAND learnt it from his Grandmother, who died a few years since, aged $82 »$ [Webster 1879 : 10]; « Laurentine, About 35 years old; learnt it from her mother $\gg$ [Ibid. : 87].

\section{La version manuscrite anglaise et le texte du livre}

On a la possibilité de comparer peu de textes anglais dans la version imprimée et manuscrite, comme on ne trouve de textes anglais que dans le premier cahier et que même tous ces contes n'ont pas été publiés. Mais on peut remarquer que la qualité de ces versions anglaises est très bonne. Donc, il s'agit d'un texte prêt à être publié. A présent, il faut comprendre comment Webster change les contes basques dans sa publication anglaise (peu de temps après avoir recueilli les contes). Dans le premier cahier du manuscrit de Webster, il y a peu de changements dans le texte anglais par rapport à celui du livre. Nous pouvons néanmoins en citer quelques-uns. Les changements des mots sont assez rares. On peut remarquer surtout des changements considérables de la ponctuation. Dans le livre, les alinéas apparaissent dans les dialogues; tandis que dans le cahier il n'y a pas d'alinéas, probablement, pour économiser du papier (nous n'indiquons pas l'absence des alinéas). Pour mieux le comprendre, on comparera quelques extraits représentatifs des versions anglaises du manuscrit avec la version imprimée de la première édition de Basque Legends. Nous citerons des morceaux de quelques contes avec des changements qui ont apparu dans le livre.

\section{THE TARTARO}

ONCE upon a time there was the son of a king who for the punishment of some fault became a monster. He could become a man again only by marrying. One day he met a young girl who refused him, because she was so frightened at him. And the Tartaro wanted to give her a ring, which she would not accept. However $\{$,$\} he sent it her by the \{a\}$ young man. As soon as the ring was upon her finger it began to say. \{,\} "Thou there, and I here\{.\}" It kept always crying out this $\{$,$\} and the Tartaro pursued her always, \{continually; \}$ and $\{$,$\} as the$ young girl had such a horror of him, she cut off the $\{$ her $\}$ finger and the ring $\{$,$\} and threw$ them into a large pond $\{$,$\} and there the Tartaro drowned himself.$

On citera encore un conte. Dans les contes plus longs il y a plus de modifications, mais elles concernent le style anglais, y compris le lexique, et ne change le sens d'aucune manière.

\section{THE BASA-JAUN. \{BASA-JAUNA, THE WILD MAN}

ONCE upon a time there $\{$ lived in a \{one\} house alady, \{the landlady\} and the farmer's wife. The farmer's wife had three sons,,$\{;\}$ one day they said to their mother to give each of them a ball and a penny roll, that they would \{wished to\} go from country to country. The mother was sorry to part with her three much $\{-\}$ loved sons- $\{;\}$ And $\{$ but $\}$ all the three started $\{$ off $\}$.

When they were in the midst of a forest they saw that night was coming on, and the eldest brother said that he would climb up the first tree. He finds a tall tree, and climbs up to the top, to the very tip-top; $\{$,$\} and the second says to him. \{:\}$

\{"\}Do you not see anything \{nothing?"\} 
He says $\{$, " $\} \mathrm{No}$, no- $\{;\}$ there's nothing to be seen, nothing; not a feather \{alone! \}- there's nothing!"

\{"\}Come down then, $\{;\}$ you are an old donkey.\{"\}

And the second climbs, and he sees nothing. The third says to him:

\{"\}You are no good at all, you others. I will climb up-\{."'\}

and $\{$ And $\}$ he climbs $\{$,$\} to the top, to the very \{$ tip\}-top. The others say to him- $\{:\}$

\{"\}And not you do \{you not\} see anything?\{"\}

He says to them, $\{:\}$

"Yes $\{;\}$ I see a long column of smoke, but very\{,\} very thin, and far, very far away. Let us go towards that, $\{$.$\} "$

and And $\}$ the three brothers set out together. At eight o'clock in the evening they come to a grand castle, and they knock at the door, and the Basa-Andre $\{$ (wild woman) $\}$ comes to answer. She asks $\{:\}$

"Who is there?"

And they reply:\{,\} "It is we who are here."

"What do you want, young children? Where are you going to at this time of night?"

"We ask and beg of you to give us shelter for to $\{-\}$ nigh- $\{;\}$ we will be satisfied with a corner of the floor, poor wretches as we are."

"I have my husband $\{$,$\} the Basa-Jaun \{$,$\} and if he catches you he will eat you- \{;\}$ that's certain."

"And if he catches us outside he will eat us all the same\{.\}"

Then she let these three brothers come in, and she hides the three in three different corners. Afterwards $\{$,$\} at nine o'clock, the Basa-Jaun comes. He made a great noise and blustering, and$ attends \{then\} the Basa-Andre goes out, and says to him:

"There is nobody here."

"Yes, you have somebody, \{;\} bring them out, or else I will eat you myself."

And she goes \{out\} and brings out the eldest brother\{,\} trembling with fright. The BasaJaun says to him-\{,\}

"Will you be my servant?"

He says to him\{,\} "Yest\{.\}"

And Basa-Jaun begins again to sniff about.

"You have still somebody else here?"

And she brings out the second, and he says to him-\{:\}

"Will you be servant to me?"

And he said $\{$,$\} "Yes."$

Again \{\} he smelled the smell of some one, and at the third time she brings out the third, and he says to him: $\{:\}$

"All three of you shall sup with me to\{-\}night, and afterwards we shall go to bed. But tomorrow we will all go hunting."

And they start on the morrow /go hunting the next dayt \{go hunting the next day\} till \{until\} eight o'clock in the evening.

Now they had at home a little sister\{.\} She was little then, but in time she grew up. One day the landlady \{and\} the farmer's wife had put out the new maize to dry in the garden fin the garden to dry $\} ;\{$.$\} And \{and\} when no one saw her, the little girl takes \{took \}$ some from her mistress' heap to \{, and $\}$ put it to her own. When the mistress saw that, she began to cry 
out $\{$,$\} saying to her- \{$,

"Bold hussey that you are, there is no one like you-\{!\} You will come to a bad end like your brothers."

And the young girl began to cry, and goes to find her mother, and says to her- $\{$,

"Mama \{Mother\}, had I any brothers?"

She says to her:\{,\} \{"\}Yes, my child."

"What were they?"

\{" $\}$ Child, they went away a long time ago, $-\{$ “ $\}$ she said to her.

This little girl says $\{$,

$\{$ [ $\} \mathrm{I}\{$,$\} too \{$,$\} must be off to-day. Give me a distaff to spin with, and a penny cake\{." \}$

She sets off, and comes to the house of the Basa Jauna \{Basa-Jaun\}, and she knocks at the door, and she lets her in. While his wife was telling her that it is the house of the asa Jauna \{Basa-Jaun\}, the eldest/r/brother/s/ comes \{the elder brother comes in\} --; but they did not recognise each other \{one another\} at all. And afterwards Basa Jauna \{Basa-Jaun\} comes, and says, as he enters the house: $\{:\}$

\{"\}You have something here for me, \{"\} says he- $\{:\}$

\{"\}No\{,"\} says she.

\{"\}Show it- $\{$. ."

and $\{$ And $\}$ immediately she shows her. Basa\{-\}Jaun says to her- $\{:\}$

"Will you engage yourself as my servant: $\{$ ?\}"

She says to him: $\{$, "YYes, sir. $\{$ " $\}$

Some days afterwards the brothers recognised their sister, and they embraced each other very much. And this young girl who was so well \{before\} began to grow thin. And one day one of her brothers asked her- $\{:\}$

"What is the matter with you that you are getting thin like that \{this\}?"

And she answered $\{:\}$

\{"\}The master every evening asks me for \{to put\} my little finger through the door\{,\} and he sucks the finger through the door, and she beeomes \{I become every day more and more stupid. \{more sad and more languid."

One day $\{$,$\} when the Basa \{-\}$ Andre was not at home $\{$,$\} these brothers and the sister plotted$ together to kill Basa\{-\}Jaun, if they could catch him in a ravine in a certain place in a ravine t diteht. And they kill him.

One day the wife asks\{,\}

"Where is Basa\{-\}Jaun?"

And Basa\{-\}Andre takes out three large teeth\{,\} and brings them to the house $\{$,$\} and tells$ this young girl herself $\{$,$\} when she beats the water for her brothers' feet in the evening \{$,$\} to put$ in each one's water a tooth \{one tooth in the water of each $\}-\{$.$\} and \{$ And $\}$ as soon as the third \{had\} finished washing the three brothers became three oxen $-\{;\}$ and this young girl used to drive all three into the fields $-\{$.$\} and \{$ And $\}$ this young girl lived there on the birds thich they (the oxen) found, and nothing else.

One day $\{$,$\} as she was passing over a bridge, she sees Basa\{-\}Andre under a bridge, and$ she says to her\{:\}

"If you do not make these three oxen men as they were before $\{$,$\} I will put you into a red-$ hot oven."

She answers her $\{:\}$ 
"No no \{No!\} go to such a dell, and take thence three hazel sticks $\{$,$\} and strike each of them$ three blows on the back.\{"\}

And she did what she told her, and they were changed into men the same as they were before, $\{;\}$ and all the brothers and the sister lived happily together in Basa\{-\}Jaun's castle, and as they lived well they made a good end also.

\{ESTEFANELLA HIRIGARAY\}

En comparant le texte du manuscrit à celui du livre, il faut ajouter qu'au lieu d'utiliser les prénoms originaux, dans son édition anglaise, Webster s'adresse à la culture traditionnelle folklorique, Yaundeko dans les contes se transforme en Juan Dekos (d'où vient, sans aucun doute, le prénom basque) et Juan de Calais dans le livre.

\section{Le choix des contes}

Dans son livre Basque Legends en anglais, Webster a publié seulement une partie de contes qu'il avait recueillis. Quels étaient, donc, ses critères de choix de contes ? Pourquoi plusieurs contes qui ont été traduits en anglais n'ont pas été publiés? (Webster mentionne souvent des « variantes inédites» dans son manuscrit).

On remarque que Cerquand, surtout dans les premières parties de ses publications des contes, utilise le critère de qualité pour trier ces contes et n'en laisse que les plus intéressants et originaux. En revanche, si Webster fait la même chose, en se fondant sur ses propres critères de ce qui est intéressant et de ce qui ne l'est pas (ainsi, il publie plutôt les contes qu'il croit être empruntés), il laisse tous les textes à la bibliothèque de Bayonne où il sait qu'ils seront bien conservés et qu'on pourra les utiliser par la suite. En plus, il sait que Julien Vinson les a recopiés. Il est probable que Webster ait décidé de ne pas publier les autres textes parce qu'il croyait que Vinson allait s'en occuper, car celui-ci était un folkloriste plus compétent. Le motif du choix des contes dans l'édition anglaise n'est pas l'originalité des textes, puisque d'après Webster, les textes folkloriques en général, ne sont pas originaux [Videgain 1974 : 454].

Dans l'édition anglaise des contes, il apparaît beaucoup de ce qu'on appelle maintenant « contes merveilleux ». Nous supposons que tout d'abord, en commençant à collecter les contes, Webster voulait attirer l'attention sur ce qui ressemblait le moins au folklore indoeuropéen. C'est pourquoi dans son premier cahier il refuse de donner les variantes des contes qui se répètent. Comme on peut le constater, ce sont les variantes les plus proches des contes occidentaux (ce n'est pas par hasard que Webster donne à l'un de ces contes un nom traditionnel The Beauty and the Beast, on ne trouve aucune différence entre le texte basque et les contes traditionnels européens ayant le même sujet). Parmi les contes qui n'ont pas été publié, les textes sont incomplets et le sujet est confus (tel est le cas de Godeon).

En comparant les recueils de Vinson et Webster, on peut remarquer qu'ils ont des critères différents pour le choix des contes. Webster est plutôt enclin à choisir des sujets communs au fonds folklorique européen, tandis que Vinson préfère les contes plus originaux, même si leur sujet reste dans une certaine mesure vague et, peut-être, incomplet. A titre d'exemple, nous pouvons citer le conte Arrosa (La rose) qui n'a pas été analysé jusqu'à maintenant. Dans ce conte, nous avons affaire avec un roi qui est parti à la guerre après avoir donné une rose à chacune de ses deux filles, en leur disant que si elles commettaient une faute, leur rose se fanerait. Toutes les deux sont séduites par un homme, et leur père voit leurs roses fanées. Ce texte correspond au début du conte dans la version de Poitou où la troisième fille se défend et 
punit le séducteur (le conte 883, d'après l'indice d'Aarne-Thompson). On trouve le texte de ce conte en anglais dans le manuscrit de Webster et dans le livre de Vinson. Pourtant, il n'a pas été publié dans Basque Legends, très probablement à cause de son caractère incomplet.

Parmi les contes qui ne sont pas choisis par Webster dans son recueil, on trouve des contes assez longs qui ressemblent beaucoup à ceux qui ont été déjà publiés, comme c'est le cas de la variante du conte Eskabi-Fidel. La seconde variante est moins complète et elle contient quelques incohérences. Un des contes, Lanterna poderosa, comme le note déjà Webster dans ses commentaires [Webster 1879 : 94], cf. aussi la remarque de Webster « cf. Aladdin's lamp et la Tabakièra » [W517v] est presque une traduction littérale d'un conte arabe d'Aladdin. On peut supposer que pour cette raison Webster ne l'a pas fait publier.

\section{Les éditions modernes}

Les textes de Webster ont été utilisés plusieurs fois dans les éditions récentes. Le texte basque de Webster a été publié en 1993 par Xipri Arbelbide qui a découvert le manuscrit. Léditeur corrige quelques fautes pour que le texte soit plus compréhensible, il ajoute également la ponctuation et modernise l'orthographe [Webster 1993 : xii-xiv], donc il s'agit plutôt d'une version qui a été faite pour les amateurs. Assez récemment, en 2005, il a paru la traduction française du recueil basé sur les notes de Webster [Webster 2005]. En 2007 un catalogue des contes merveilleux qui contient plusieurs contes de Webster est paru au Pays Basque espagnol [Etxeberria 2007]. Quelques contes du recueil de Webster ont été publiés par Charles Videgain et Angel Irigarai à la fin des années 70 dans Fontes Lingvae Vasconvm (« Antes de ahora no ha sido examinado este inédito manuscrito, al parecer, por nadie » [Irigarai, Videgain 1974 : 451]).

En ce qui concerne le fonctionnement du manuscrit et les éditions différentes du texte, l'une des plus intéressantes a été l'édition française de Basque Legends parue récemment, en 2005 chez Aubéron [Webster 2005] (la traduction espagnole du recueil est faite à partir de l'édition anglaise de Basque Legends, donc elle est moins intéressante d'un point de vue textologique, et la version basque du texte publiée en 1993 reste assez fidèle à l'original). On insiste qu'il s'agit de l'édition française du texte anglais, puisque le traducteur (Nicolas Burguète) conserve les chapitres et les contes que Webster a choisi, bien qu'il existe plusieurs autres contes qui n'ont pas paru qu'en basque en 1993 et dont la traduction française et anglaise existe dans le manuscrit du folkloriste. Pourquoi décide-t-il de respecter le choix de Webster ? On peut supposer que l'éditeur a eu l'intention de publier une correspondance plus ou moins exacte du recueil de Webster et remplir la lacune qui avait existé jusqu'à la publication (le texte de Basque Legends en français), ce que prouve la remarque du traducteur : « depuis leur première édition à Londres, en 1877, les Basque Legends de révérend Webster n'avaient jamais été traduites en français dans leur intégralité » [Ibid. : 16]. Donc, la traduction française du texte est tout d'abord un hommage au folkloriste célèbre, tandis que l'édition basque des contes du manuscrit a été créée comme un monument du folklore basque. Cependant, en traduisant le texte en français, Nicolas Burguète utilise dans une grande mesure les notes de Webster (et pas les traductions de Vinson qui sont souvent plus correctes). En comparant les deux textes, on peur affirmer qu'en réalité il s'agit plutôt de la traduction faite à l'aide de madame Bellevue, écrite par Webster et corrigée par Burguète, malgré la remarque « Traduction, recherches et notes de Nicolas Burguète» [Ibid. : 5] (qui confesse, cependant, que la traduction française « s'appuie sur les notes manuscrites de l'auteur» [Ibid. : 17]). Pourtant, on ne pense pas 
que cette remarque suffise pour que le lecteur du recueil en français comprenne dans quelle mesure la traduction reste fidèle au manuscrit de Webster.

A titre d'exemple on va citer deux courts passages du même conte de Wesbster (Le Merle blanc) tirés du manuscrit de Webster et du recueil en français.

Le texte du manuscrit [W411v]:

«Comme beaucoup dans le monde il y avait un roi qui avait trois fils. Ce roi était aveugle et il avait appris un jour qu'il y avait un roi qui avait un merle blanc lequel donnait la vue aux aveugles. Le fils aîné quand il entendit cela dit à son père qu'il irait lui au plus vite chercher ce merle. Le père lui dit Je préfère rester aveugle que de me séparer de /toi/ cet enfant. Le fils lui dit n'ayez aucune crainte de moi avec un cheval chargé d'argent qu'il trouverait et porterait le merle. »

Les Légendes Basques en français [Webster 2005: 213] :

«Comme beaucoup dans le monde, il y avait un roi qui avait trois fils. Ce roi était aveugle et il avait appris un jour qu'il y avait un roi qui avait un merle blanc, qui rendait la vue aux aveugles. Quand son fils aîné entendit cela, il dit à son père qu'il irait lui chercher ce merle blanc au plus vite.

Le père lui dit : « Je préfère rester aveugle que de me séparer de toi, mon enfant.

Le fils lui dit : n'ayez aucune crainte pour moi, avec un cheval chargé d'argent je le trouverai et vous l'apporterai».

Tout d'abord, Burguète corrige l'orthographe et la ponctuation de Webster (si les traductions anglaises de Webster sont d'une très haute qualité, la plupart des traductions français ne le sont pas), les fautes de français et l'emploi du discours direct et indirect. Par contre, le traducteur conserve la division en alinéas de la traduction anglaise de Basque Legends.

Burguète utilise d'un côté, le texte basque et français du manuscrit, et de l'autre côté, le texte anglais publié par Webster, cf. les mots entre parenthèses qui manquent dans le texte basque et que Webster reconstitue pour rendre le texte plus compréhensible : «Ezagutu zuen neskatcha hua zela erakutsi zion kiloa eta lardatza » [W47], « (Then) he recognised the young girl. She showed him (too) the distaff and spindle » [Webster 1879: 41] et «(Alors) il reconnut la jeune fille. Elle lui montra (aussi) la quenouille et le fuseau » [Webster 2005 : 77] ; « lehenago banuen armario batentzat gakhoat eta hura galdu nuen eta inaazi nuen berri bat. Eta errantzion andriari » [W47], «Formerly, I had a key to a chest of drawers, and I lost it, and had a new one made. (After that, I found the old one.) And he turned to his wife » [Webster 1879: 41] vs. « Autrefois, j'avais une clef pour une armoire, je l'ai perdu et en ai fait une neuve. (Puis, j'ai retrouvé l'ancienne.) Et il se tourna vers sa femme» [Webster 2005 : 77]. Dans le dernier morceau, le verbe «tourner» au lieu de « dire », comme en basque, prouve l'influence du texte anglais.

Le recueil de Webster a été traduit en d'autres langues. L'existence de la traduction espagnole [Webster 1989] est surtout intéressante puisque c'est la quatrième version successive du texte original : Tout d'abord le texte a été enregistré en basque et réécrit au moins une fois, puis traduit en français à l'aide de Mme Bellevue, puis en anglais par Webster, et enfin en espagnol.

En conclusion, on voudrait remarquer que jusqu'en 2007 le lecteur non bascophone n'a pas eu accès qu'aux contes que Webster a choisi pour être publiés en 1877 et la seconde édition de 1879. Nicolas Burguète qui traduit les contes à partir du manuscrit de Webster, ne reprend que ces contes-là. Bien qu'il appelle son ouvre « une traduction attendue depuis un 
siècle » [Webster 2005 : 16] et écrive que « Depuis leur première édition à Londres, en 1877 ; les Basque Legends de révérend Webster n'avaient jamais été traduites en français dans leur intégralité », son propre livre n'est pas intégral : une moitié de contes n'y a pas été publiée. En 2007 plusieurs contes publiés dans le recueil de Xipri Arbelbide ont été traduits en espagnol [Etxeberria 2007]. Il serait souhaitable de les traduire en d'autres langues européennes, surtout en français et en anglais.

Les manuscrits de Webster et de Vinson nous donnent également plusieurs pistes de recherche : il est indispensable d'analyser les manuscrits de Webster qui se trouvent à la Bodleian Library (Université d'Oxford) ; il faudrait essayer de trouver des traces des informants de Webster ; il est également nécessaire d'effectuer la recherche sur l'édition française de contes qui n'a pas été publiée.

\section{Bibliographie}

1. AbBadie A. Th. de. Varia Linguistica et litteraria // Hizkuntza eta Literatura. Recueil de textes ethnographiques, géodésiques, linguistiques, littéraires. Donostia-San Sebastián: EuskoIkaskuntza, Euskaltzaindia, 1997, pp. 351-456.

2. Boggs R. S. Index of Spanish folktales, classified according to Antti Aarne's Types of the folktale, translated and enlarged by Stith Thompson // FF communications 74, 1930. Chicago. The University of Chicago, 1930.

3. Cerquand J.-F. Légendes E Récits Populaires du Pays basque // Société de sciences, lettres et arts de Pau. 1876-1877. II série. Tome 6me, pp. 450-531.

4. Cerquand J.-F. Légendes E Récits Populaires du Pays basque // Société de sciences, lettres et arts de Pau. 1882-1883. II série. Tome 11me, pp. 101-294.

5. CuzAcQ R. Le dragon de Lissarague et sa légende à Saint-Pierre d'Irube // Société des sciences, lettres et arts de Bayonne, 131, 1975, pp. 191-201.

6. Echegaray Corta C. de. Wenworth Webster // RIEV, 2, 1908, pp. 373-385.

7. Espagne M. De l'archive au texte. Recherches d'histoire génétique. Paris : PUF, 1998.

8. ETXEBERRIA M. Ipuin magikoak. Herri kultiraren narrazioak (I, II, III). Ipuin erromantikoak. Herri kultiraren narrazioak (IV). Lasarte-Oria: Etor-Ostoa, 2007.

9. Irigarai A, Videgain Ch. Noticias bio-bibliográficas del vascólogo y etnólogo Wentworth Webster // Fontes Lingva Vasconvm. Studia et documenta. Pamplona, 18, 1974, pp. 451464.

10. Jasmin N. Naissance du conpe féminin: Madame d'Aulnoy // Madame d'Aulnoy. Contes de fées. Edition critique établie par Nadine Jasmin. Paris : Honoré Champion, 2008, pp. $9-75$.

11. Lacombe, Georges. Julien Vinson // Gure Herria. Janvier-Février 1927, pp. 60-64.

12. Lacombe G. Wentworth Webster // RIEB, 3. Mai 1907, Paris: Paul Geuthner, p. 323.

13. Lafitte P. Grammaire basque (Navarro-Labourdin littéraire). Edition revue et corrigée. Donostia : Elkarlanean, 2001.

14. Lebrave J.-L. Du visible au lisible : comment représenter la genèse // Genesis 27/06, . 1118.

15. Mercero I. Websterren ipuinak. Hurbilketa analitikoa // Ipuin Erromantikoak IV eta Websterren Ipuinak Hurbilketa analitikoa. Herri kulturaren narrazioak (31), 2007, pp. 1-111. 
16. Urkizu P. Wentworth Webster. Eusko-Jarlaritza. 19 sorta. Vitoria-Gasteiz, 2000.

17. URquijo J. de. Julien Vinson // RIEV, 18, 1927, pp. 217-224.

18. URQuijo J. de. Vascófilos ingleses. A propósito de "Un libro de los vascos" de Rodney Gallop // RIEV, , 1935, pp. 201-224.

19. VeYRIN P. Les Basques de Labourd, de Soule et de Basse-Navarre, leur histoire et leurs traditions. Grenoble: Arthaud, 1955.

20. VeYRIn P. Lettres du Prince Louis Lucien Bonaparte à Wentworth Webster // RIEV, 25, 1934, pp. 316-333.

21. Videgain, Charles. Quelques contes basques tirés du manuscrit Webster // Fontes Lingvae Vasconvm. Studia et documenta. Pamplona, 18, 1974, pp. 453-464.

22. Vinson J. Basque Legends. Manuscrit. (MS 720, Bibliothèque Municipale de Bayonne).

23. Vinson J. Bibliographie du folk-lore basque [1] // Société des sciences, lettres et arts de Bayonne, 1884.

24. Vinson J. Le folklore du Pays Basque. Paris: G.-P. Maisonneuve \& Larose, Editeurs, 1883.

25. Vinson J. Les Basques et le Pays basque. Mœurs, langage et histoire. Nîmes : Lacour -

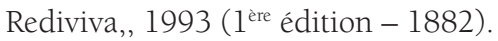

26. Vinson. Manuel de la langue Hindustani (Urdû et Hindî). Grammaire, textes, vocabulaires. Paris : Maisonneuve, 1899.

27. Vinson Julien. <Une lettre> // Gure Herria, 111921.

28. Webster W. Basque Legends. Manuscrit. (MS 88, Bibliothèque Municipale de Bayonne).

29. Webster W. Basque Legends: Collected, Chiefly in the Labourd, by Rev. Wentworth Webster, M. A., Oxon. With an Essay on the Basque Language, by M. Julien Vinson, of the Revue de Linguistique, Paris together with Appendix: Basque Poetry London: Griffith and Farran, Successors to Newbery and Harris, Corner of St. Paul's Churchyard; and Walbrook \& co., 52, fleet street, e.c. [1879].

30. Webster W. Ipuinak. Xipri Arbelbideren edizioa. Donostia : Klasikoak, 1993.

31. Webster W. Leyendas vascas. Madrid : Miraguano Ediciones, 1989.

32. Webster W. Légendes basques. Recueillis principalement dans la province du Labourd. Edition Auberon. 2005.

33. Webster W. Légendes E récits populaires du Pays basque par M. Cerquand, Inspecteur de l’Académie de Bordeaux // Bulletin de la société Ramon. Octobre, 1875, pp. 167-174.

34. ЛихАчев Д. С. Текстология: на материале русской литературы X-XVII вв. / при участии А. А. Алексеева и А. Г. Боброва; РАН, Институт русской литературы (Пушкинский Дом). - 3-е изд., перераб. и доп. - СПб.: Алетейя, 2001 (Likhachev D. S. Textologie). 